\title{
Theoretical and computational comparison of models for dislocation dissociation and stacking fault / core formation in fcc crystals
}

\author{
J. R. Mianroodi \\ Material Mechanics, RWTH Aachen, Aachen, D-54062, Germany \\ A. Hunter, I. J. Beyerlein \\ Los Alamos National Laboratory, P.O. Box 1663 MS T086, Los Alamos, NM 87545, USA \\ B. Svendsen \\ Material Mechanics, RWTH Aachen, Aachen, D-54062, Germany \\ Microstructure Physics and Alloy Design, \\ Max-Planck-Institut für Eisenforschung GmbH, D-40237 Düsseldorf, Germany
}

\begin{abstract}
The purpose of the current work is the theoretical and computational comparison of selected models for the energetics of dislocation dissociation resulting in stacking fault and partial dislocation (core) formation in fcc crystals as based on the (generalized) Peierls-Nabarro (GPN: e.g., Xiang et al., 2008; Shen et al., 2014), and phase-field (PF: e.g., Shen and Wang, 2004; Hunter et al., 2011, 2013; Mianroodi and Svendsen, 2015), methodologies (e.g., Wang and Li, 2010). More specifically, in the current work, the GPN-based model of Xiang et al. (2008) is compared theoretically with the PF-based models of Shen and Wang (2004), Hunter et al. (2011, 2013), and Mianroodi and Svendsen (2015). This is carried out here with the help of a unified formulation for these models via a generalization of the approach of Cahn and Hilliard (1958) to mechanics. Differences among these include the model forms for the free energy density $\psi_{\text {ela }}$ of the lattice and the free energy density $\psi_{\text {sli }}$ associated with dislocation slip. In the PF-based models, for example, $\psi_{\text {ela }}$ is formulated with respect to the residual distortion $\boldsymbol{H}_{\mathrm{R}}$ due to dislocation slip (e.g., Khachaturyan, 1983; Mura, 1987), and with respect to the dislocation tensor curl $\boldsymbol{H}_{\mathrm{R}}$ in the GPN model (e.g., Xiang et al., 2008). As shown here, both model forms for $\psi_{\text {ela }}$ are in fact mathematically equal and so physically equivalent. On the other hand, model forms for $\psi_{\text {sli }}$ differ in the assumed dependence on the phase or disregistry fields $\phi$, whose spatial variation represents the transition from unslipped to slipped regions in the crystal. In particular, Xiang et al. (2008) and Hunter et al. $(2011,2013)$ work with $\psi_{\text {sli }}(\phi)$. On the other hand, Shen and Wang (2004) and Mianroodi and Svendsen (2015) employ $\psi_{\text {sli }}(\phi, \nabla \phi)$.
\end{abstract}

To investigate the consequences of these differences for the modeling of the dislocation core, dissociation, and stacking fault formation, predictions from the models of Hunter et al. (2011, 
2013) and Mianroodi and Svendsen (2015) are compared with results from molecular statics (MS) for the deformation field of dissociated edge and screw dipoles in $\mathrm{Al}$ and Au. Particularly notable is the agreement of the MS and PF strain field results for the case of perfect screw dissociation which, in contrast to the edge case, are characterized by asymmetric displacement and strain fields. The degree of this asymmetry is apparently related to the corresponding anisotropy ratio. As well, comparison of MS and PF disregistry fields implies that the gradient dependence of $\psi_{\text {sli }}$ results in a broadening of the (otherwise too narrow) disregistry profile to the form predicted by MS.

Keywords: phase field, dislocation, dissociation, core, stacking fault, molecular statics

\section{Introduction}

Generally speaking, the energetically favored state of a dislocation line or loop in close-packed metals is its dissociated state, consisting of leading and trailing partial dislocation (cores) bounding, and separated by, a stacking fault (SF). Specific characteristics of this configuration such as SF width are dynamic in nature and depend on external loading conditions. In general, the details of core and SF formation play a role in a number of other processes such as (core) reactions, cross slip, structural transformation (e.g., martensite formation), twinning, or network formation (e.g., Hirth and Lothe, 1982).

In the last decade or so, a number of continuum models for the energetics and dynamics of dislocation and defect processes on atomic length-scales and over diffusive timescales have been developed with the help of Peierls-Nabarro (PN: e.g., Schoeck, 2005) and phase-field (PF: e.g., Bulatov and Cai, 2006; Wang and Li, 2010) approaches. Particular models of the PN-type include Xiang et al. (2008), Wang (2008), Wang et al. (2011), Shen et al. (2014) and Wang (2015), while Shen and Wang (2004), Hunter et al. (2011, 2013) and Mianroodi and Svendsen (2015) represent models of the PF-type. Apparent differences in PN- and PF-type models include the treatment of elastic interactions, the energetics of dislocation states (e.g., the dissociated state), and the modeling of the dislocation core.

In order to investigate and understand these differences in more detail, a theoretical and computational comparison of selected PN- and PF-based dislocation models is carried out in the current work. In particular, on the theoretical side, the generalized PN (GPN) model of Xiang et al. (2008), the PF dislocation dynamics (PFDD) model of Hunter et al. (2011, 2013), and the PF-based models of Shen and Wang (2004) and Mianroodi and Svendsen (2015), are compared. To facilitate this, the PF-based models are formulated in the current work in a unified fashion via generalization of the original approach of Cahn and Hilliard (1958) and Allen and Cahn (1979) to mechanical systems. In the second part of the work, computational and simulation results based on the models of Hunter et al. (2011, 2013) and Mianroodi and Svendsen (2015) are compared with each other and with molecular statics results for dislocation dissociation, SF and core formation in fcc Au and Al. For comparability, both models are identified using the same set of atomistic data for lattice and elastic properties, $\gamma$-surfaces, and dislocation cores, obtained from the embedded atom method (EAM) potentials for Al (Mishin et al., 1999) and Au (Grochola et al., 2005) in the molecular statics simulations. Consequently, any difference in results is due solely to model differences. 
The work begins in Section 2 with the unified PF-model formulation and generalization of the original approach of Cahn and Hilliard (1958) and Allen and Cahn (1979) to mechanical systems in the context of geometric linearity. This is complemented by a detailed unified treatment of the elastic energy and stress models employed in GPN- and PF-based models in Appendix A, and the GPN-based modeling of the SF energy (SFE) in Appendix B. Given these theoretical results, attention turns in the remainder of the work to the comparison of computational and simulation results for $\mathrm{Al}$ and $\mathrm{Au}$ based on molecular statics and PF modeling. To this end, the PF models are identified in Section 3 with the help of atomistic data obtained from the interatomic potentials used in the molecular statics simulations. With the help of the identified models, a number of comparison of simulation results are then discussed in Section 4. The paper ends in Section 5 with a summary and discussion. Details concerning the algorithmic formulation of the PF models are provided in Appendix C.

Before we begin, a word on notation. In this work, (three-dimensional) Euclidean vectors are represented by lower-case bold italic characters $\boldsymbol{a}, \boldsymbol{b}, \ldots$, In particular, let $\boldsymbol{i}_{x}, \boldsymbol{i}_{y}, \boldsymbol{i}_{z}$ represent the Cartesian basis vectors. Second-order tensors are represented by upper-case bold italic characters $\boldsymbol{A}, \boldsymbol{B}, \ldots$, with $\boldsymbol{I}$ the second-order identity. By definition, any $\boldsymbol{A}$ maps any $\boldsymbol{b}$ linearly into a vector $\boldsymbol{A} \boldsymbol{b}$. Fourth-order Euclidean tensors $\boldsymbol{A}, \boldsymbol{B}, \ldots$, are denoted by upper-case slanted sans-serif characters; by definition, any $\boldsymbol{A}$ maps any $\boldsymbol{B}$ linearly into a second-order tensor $\boldsymbol{A} \boldsymbol{B}$. Let $\mathcal{A} \cdot \mathcal{B}=A_{i \ldots} B_{i \ldots}$ represent the scalar product of two arbitrary-order tensors $\mathcal{A}$ and $\mathcal{B}$. Using this, $\boldsymbol{A}^{\mathrm{T}} \boldsymbol{b} \cdot \boldsymbol{c}:=\boldsymbol{b} \cdot \boldsymbol{A} \boldsymbol{c}$ defines the transpose $\boldsymbol{A}^{\mathrm{T}}$ of $\boldsymbol{A}$, and $\boldsymbol{A}^{\mathrm{T}} \boldsymbol{B} \cdot \boldsymbol{C}:=\boldsymbol{B} \cdot \boldsymbol{A} \boldsymbol{C}$ that $\boldsymbol{A}^{\mathrm{T}}$ of $\boldsymbol{A}$. Let sym $\boldsymbol{A}:=\frac{1}{2}\left(\boldsymbol{A}+\boldsymbol{A}^{\mathrm{T}}\right)$ represent the symmetric part of $\boldsymbol{A}$. Additional notation will be introduced as needed in what follows.

\section{Unified PF-based model formulation \& theoretical comparison}

As discussed in the introduction, the PF-based models of Shen and Wang (2004), Hunter et al. (2011, 2013), and Mianroodi and Svendsen (2015), can all be formulated in the context of a direct extension of the formulation of Cahn and Hilliard (1958) for inhomogeneous chemical systems to mechanics. Let $\boldsymbol{u}$ represent the displacement field, $\boldsymbol{H}=\nabla \boldsymbol{u}$ the distortion field, $\boldsymbol{E}=\operatorname{sym} \boldsymbol{H}$ the strain field, and $\boldsymbol{\phi}=\left(\phi_{1}, \ldots, \phi_{n}\right)$ a set of $n$ phase fields. The relevant generalization

$$
\psi(\boldsymbol{E}, \boldsymbol{\phi}, \nabla \boldsymbol{\phi}, \nabla \nabla \boldsymbol{\phi}, \ldots)
$$

of the general free energy density of Cahn and Hilliard (1958) to mechanics is restricted here for simplicity to a dependence on $\boldsymbol{E}$ alone. Strain-gradient-based or even higher-order mechanical extensions are also possible and may even be necessary to be consistent with recent DFT-based results (Iyer et al., 2015) for $\mathrm{Al}$ which predict significant strain gradients in the core. Following Cahn and Hilliard (1958) then, consider a generalized Taylor series expansion of the general free energy density about the (with respect to $\phi$ ) homogeneous energy state

$$
\left.(\boldsymbol{E}, \boldsymbol{\phi}, \nabla \boldsymbol{\phi}, \nabla \nabla \boldsymbol{\phi}, \ldots)\right|_{\text {hom }}:=\left(\boldsymbol{E}_{\mathrm{R}}(\boldsymbol{\phi}), \boldsymbol{\phi}, \mathbf{0}, \mathbf{0}, \ldots\right)
$$

depending in particular on the zero-stress or "residual" strain $\boldsymbol{E}_{\mathrm{R}}$, i.e., $\left.\partial_{\boldsymbol{E}} \psi\right|_{\text {hom }}=\mathbf{0}$. As indicated, $\boldsymbol{E}_{\mathrm{R}}$ itself depends on $\boldsymbol{\phi}$ in the current context (see (7) below). In the (non-polar) centrosymmetric case, $\left.\partial_{\nabla \phi_{a}} \psi\right|_{\text {hom }}=\mathbf{0}(a=1, \ldots, n)$ identically. Further, attention is restricted here for simplicity 
to isotropic gradient ${ }^{1}$ energy. Expansion of $\psi$ to second-order and neglect of all mixed terms then results in the approximation

$$
\begin{aligned}
\psi(\boldsymbol{E}, \boldsymbol{\phi}, \nabla \boldsymbol{\phi}, \nabla \nabla \boldsymbol{\phi}, \ldots) & \approx \frac{1}{2}\left(\boldsymbol{E}-\boldsymbol{E}_{\mathrm{R}}\right) \cdot \boldsymbol{C}_{\mathrm{E}}\left(\boldsymbol{E}-\boldsymbol{E}_{\mathrm{R}}\right) \\
& +\left.\psi\right|_{\mathrm{hom}}+\left.\sum_{a=1}^{n} \partial_{\nabla \nabla \phi_{a}} \psi\right|_{\mathrm{hom}} \cdot \nabla \nabla \phi_{a}+\left.\frac{1}{2} \sum_{a, b=1}^{n} \nabla \phi_{a} \cdot \partial_{\nabla \phi_{a}} \partial_{\nabla \phi_{b}} \psi\right|_{\mathrm{hom}} \nabla \phi_{b}
\end{aligned}
$$

in terms of the moduli $C_{\mathrm{E}}:=\left.\partial_{E} \partial_{E} \psi\right|_{\text {hom }}$. Further,

$$
\left.\sum_{a=1}^{n} \partial_{\nabla \nabla \phi_{a}} \psi\right|_{\mathrm{hom}} \cdot \nabla \nabla \phi_{a}+\left.\frac{1}{2} \sum_{a, b=1}^{n} \nabla \phi_{a} \cdot \partial_{\nabla \phi_{a}} \partial_{\nabla \phi_{b}} \psi\right|_{\mathrm{hom}} \nabla \phi_{b}=\frac{1}{2} \sum_{a, b=1}^{n} \nabla \phi_{a} \cdot \boldsymbol{\Lambda}_{a b} \nabla \phi_{b}
$$

for pure bulk systems or no-flux boundary conditions $\left.\partial_{\nabla \nabla \phi_{a}} \psi\right|_{\text {hom }} \nabla \phi_{a} \cdot \boldsymbol{n}=0$. Here, $\boldsymbol{\Lambda}_{a b}:=$ $\left.\partial_{\nabla \phi_{a}} \partial_{\nabla \phi_{b}} \psi\right|_{\text {hom }}-2 \partial_{\phi_{a}}\left(\left.\partial_{\nabla \nabla \phi_{b}} \psi\right|_{\text {hom }}\right)$. Restricting attention to (lattice- / glide-plane orientation preserving) dislocation glide in single crystals, $C_{\mathrm{E}}$ and $\boldsymbol{\Lambda}_{a b}$ are treated as constant here for simplicity. Then (3) reduces to the model form

$$
\psi(\boldsymbol{E}, \boldsymbol{\phi}, \nabla \boldsymbol{\phi})=\psi_{\mathrm{ela}}(\boldsymbol{E}, \boldsymbol{\phi})+\psi_{\mathrm{hom}}(\boldsymbol{\phi})+\psi_{\mathrm{gra}}(\nabla \boldsymbol{\phi})
$$

for $\psi$, with

$$
\begin{aligned}
\psi_{\text {ela }}(\boldsymbol{E}, \boldsymbol{\phi}) & :=\frac{1}{2}\left(\boldsymbol{E}-\boldsymbol{E}_{\mathrm{R}}(\boldsymbol{\phi})\right) \cdot \boldsymbol{C}_{\mathrm{E}}\left(\boldsymbol{E}-\boldsymbol{E}_{\mathrm{R}}(\boldsymbol{\phi})\right), \\
\psi_{\mathrm{hom}}(\boldsymbol{\phi}) & :=\left.\psi\right|_{\mathrm{hom}}\left(\boldsymbol{E}_{\mathrm{R}}(\boldsymbol{\phi}), \boldsymbol{\phi}\right), \\
\psi_{\mathrm{gra}}(\nabla \boldsymbol{\phi}) & :=\frac{1}{2} \sum_{a, b=1}^{n} \nabla \phi_{a} \cdot \boldsymbol{\Lambda}_{a b} \nabla \phi_{b},
\end{aligned}
$$

the elastic, "homogeneous", and "gradient", parts of $\psi$, again in the context of the PF approach. In particular, $\psi_{\text {ela }}$ is just the Khachaturyan-Shalatov microelastic relation (e.g., Khachaturyan, 1983; Wang et al., 2001; Wang and Li, 2010) based on residual strain. As is shown in detail in Appendix A, the form of $\psi_{\text {ela }}$ in (6) is consistent with the PF-based models of Hunter et al. (2011, 2013) and Mianroodi and Svendsen (2015), as well as with the GPN models of Xiang et al. (2008) and Shen et al. (2014). Common to both the PF- and GPN-based models is the association of $\psi_{\text {hom }}$ with SFs and the corresponding generalized SF energy $\psi_{\text {sfe }}$. In addition, the models of Xiang et al. (2008), Hunter et al. (2011, 2013), and Shen et al. (2014) associate $\psi_{\text {sli }}$ with $\psi_{\text {hom }}$ and so with $\psi_{\text {sfe }}$. On the other hand, in Shen and Wang (2004) and Mianroodi and Svendsen (2015), $\psi_{\text {sli }}$ is associated with $\psi_{\text {hom }}+\psi_{\text {gra }}$, the former being again $\psi_{\text {sfe }}$, and the latter associated with (partial) dislocation lines / cores where $\nabla \boldsymbol{\phi}$ is maximal. This distinction was basically established early on in the work of Shen and Wang (2004), who examined the effect of varying the gradient-energy coefficient $\psi_{\mathrm{G} 0}$ in (8) on dissociation and core formation in Pd. They showed that this coefficient affects primarily the core (e.g., size) of the dislocation (partial or perfect), and to a lesser extent, the SF width, which is primarily affected by the SFE and elastic interaction.

\footnotetext{
${ }^{1}$ As discussed recently by Nani and Gururajan (2014), in general, an explicit dependence of $\psi$ on second-order $\nabla \nabla \phi$ and third-order $\nabla \nabla \nabla \phi$ gradients, and the corresponding fourth- and six-order tensor moduli, are required for cubic or hexagonal (i.e., anisotropic) gradient energy. For isotropic systems as well as symmetries such as tetragonal, second-rank tensor gradient moduli suffice.
} 
For the case of dislocation slip, the residual distortion $\boldsymbol{H}_{\mathrm{R}}$ determining $\boldsymbol{E}_{\mathrm{R}}=\operatorname{sym} \boldsymbol{H}_{\mathrm{R}}$ takes the corresponding simple shear form

$$
\boldsymbol{H}_{\mathrm{R}}(\boldsymbol{\phi})=\sum_{a=1}^{n} \eta_{a}\left(\phi_{a}\right) \boldsymbol{s}_{a} \otimes \boldsymbol{n}_{a}
$$

(e.g., Khachaturyan, 1983; Mura, 1987; Bulatov and Cai, 2006). Here, $\eta_{a}$ is amount of (shear) slip from system $a$ in the direction $\boldsymbol{s}_{a}:=\boldsymbol{b}_{a} / b_{a}$ on the plane with unit normal $\boldsymbol{n}_{a}$. As usual, $b_{a}:=\left|\boldsymbol{b}_{a}\right|$ is the magnitude of the corresponding Burgers vector $\boldsymbol{b}_{a}$. Shen and Wang (2004) and Hunter et al. $(2011,2013)$ worked with $\phi_{a}:=\eta_{a} / b_{a}$ and 3 in-plane systems per $\{111\}$ plane, i.e., one for each $\langle 110\rangle$ direction, such that $n=12$. On the other hand, Xiang et al. (2008) and Mianroodi and Svendsen (2015) worked with 2 in-plane systems, i.e., one perfect $\langle 110\rangle$, and one partial $\langle 112\rangle$, per $\{111\}$ plane (e.g., Bulatov and Cai, 2006, Chapter 10), in which case $n=8$. In particular, Mianroodi and Svendsen (2015) assumed $\phi_{a}:=\eta_{a} / \gamma_{a}$. Here, $\gamma_{a}:=b_{a} / d_{a}, d_{a}=d_{111}=a_{0} / \sqrt{3}$ is the $\{111\}$ interplanar spacing, and $a_{0}$ is the lattice constant. Each $\phi_{a}$ models the transition between different states of slip in the energy landscape of the single crystal (more on this below). These fields are modeled such that $\boldsymbol{H}_{\mathrm{R}}$ vanishes everywhere in the material except on slipped surfaces, i.e., has support only on such surfaces.

Since the current work is restricted to dislocation slip, the dependence of $\psi_{\text {gra }}$ on $\nabla \phi$ at the single crystal level can be modeled by the Nye measure curl $\boldsymbol{H}_{\mathrm{R}}$. In particular, this measure projects $\nabla \boldsymbol{\phi}$ onto the slip systems. On this basis, the pure-glide-based form

$$
\psi_{\text {gra }}(\nabla \boldsymbol{\phi})=\psi_{\mathrm{G} 0}\left|\operatorname{curl} \boldsymbol{H}_{\mathrm{R}}\right|^{2}
$$

for $\psi_{\text {gra }}$ in (6) was adopted by Mianroodi and Svendsen (2015), in which case

$$
\boldsymbol{\Lambda}_{a b}=\psi_{\mathrm{G} 0} \boldsymbol{N}_{a b}^{g}, \quad \boldsymbol{N}_{a b}^{g}:=\gamma_{a} \gamma_{b}\left(\boldsymbol{s}_{a} \cdot \boldsymbol{s}_{b}\right)\left\{\left(\boldsymbol{n}_{a} \cdot \boldsymbol{n}_{b}\right) \boldsymbol{I}-\boldsymbol{n}_{b} \otimes \boldsymbol{n}_{a}\right\},
$$

follows via (7). Note that the scaling factor $\psi_{\mathrm{G} 0}$ has unit of energy per unit length. If interactions among distinct slip systems $(a \neq b)$ are neglected, $(8)$ reduces to the model considered for example by Bulatov and Cai (2006, Equation (11.34)).

The basic model formulation is completed in all PF-based models as usual by quasi-static mechanical equilibrium

$$
\operatorname{div} \boldsymbol{T}=\mathbf{0}
$$

in terms of the linear elastic stress

$$
\boldsymbol{T}=\partial_{\boldsymbol{E}} \psi=C_{\mathrm{E}}\left[\boldsymbol{E}-\boldsymbol{E}_{\mathrm{R}}\right]
$$

as well as the time-dependent Ginzburg-Landau (TDGL) relation

$$
\dot{\phi}_{a}=m_{0}\left(\operatorname{div} \partial_{\nabla \phi_{a}} \psi-\partial_{\phi_{a}} \psi\right)
$$

for $\phi_{1}, \ldots, \phi_{n}$. For the current case of an infinite periodic medium, (10) is subject to stress-based boundary conditions, and (12) to periodic no-flux boundary conditions (see Appendix C for more 
details). Non-negative mobility $m_{0} \geqslant 0$ (assumed constant and equal for all $a=1, \ldots, n$ for simplicity) insures non-negative dissipation and gradient flow of the system toward thermodynamic equilibrium.

Besides the results from Appendix A for the elastic energy, this completes the theoretical comparison. In the rest of the work, attention is focused on the computational comparison of results from the models of Hunter et al. (2011, 2013) and Mianroodi and Svendsen (2015) with each other and analogous results from molecular statics (MS) for the dissociation of perfect edge and screw dipoles in $\mathrm{Al}$ and $\mathrm{Au}$. To this end, these models are first identified for these materials using the same data set obtained from interatomic potentials.

\section{Model identification for $\mathrm{Au}$ and $\mathrm{Al}$}

\subsection{Basic data and elastic energy $\psi_{\text {ela }}$}

The PF models are identified with the help of data from EAM potentials for Al (Mishin et al., 1999) and Au (Grochola et al., 2005). Basic lattice and elastic properties for these are summarized in Table 1.

\begin{tabular}{|c|c|c|c|c|}
\hline & $a_{0}[\AA]$ & $C_{11}[\mathrm{GPa}]$ & $C_{12}[\mathrm{GPa}]$ & $C_{44}[\mathrm{GPa}]$ \\
\hline $\mathrm{Au}$ & 4.08 & 201.65 & 169.53 & 45.97 \\
\hline $\mathrm{Al}$ & 4.05 & 113.80 & 61.55 & 31.60 \\
\hline
\end{tabular}

Table 1: Lattice parameter $a_{0}$ and elastic constants $C_{11}, C_{12}, C_{44}$ calculated from EAM potentials for Al (Mishin et al., 1999) and Au (Grochola et al., 2005).

Given these, $d_{111}=a_{0} / \sqrt{3}, b_{110}=a_{0} / \sqrt{2}$, and $b_{112}=a_{0} / \sqrt{6}$, as well as $\gamma_{a}, \boldsymbol{E}_{a}$ for $a=1, \ldots, n$, and $\boldsymbol{H}_{\mathrm{R}}$, are determined. The elastic constants determining $\boldsymbol{C}_{\mathrm{E}}$, and so $\psi_{\text {ela }}$, are then identified for $\mathrm{Al}$ and $\mathrm{Au}$.

\subsection{Stacking fault energy $\psi_{\text {sfe }}$}

To obtain atomic data for the (generalized) stacking fault energy (SFE) based on the EAM potentials, a simulation box containing 2700 atoms was subject to 200 displacement steps in the [110] direction and 600 displacement steps in the [1̄12] direction. After each such step, the system is relaxed normal to the glide plane and the resulting energy per unit area $\gamma_{\text {sfe }}\left(\phi_{\overline{1} 10}, \phi_{\overline{1} \overline{1} 2}\right)$ relative to the ideal fcc lattice was calculated as a function of $\phi_{1}=\phi_{\overline{1} 10}$ and $\phi_{2}=\phi_{\overline{1} \overline{1} 2}$.

Using such atomic data, Mianroodi and Svendsen (2015) employed a simple numerical scheme to determine $\psi_{\text {sfe }}\left(\phi_{1}, \phi_{2}\right)$ and its phase-field derivatives. These were then used in look-up-table fashion for the simulations. Alternatively, Hunter et al. $(2011,2013)$ fitted the truncated Fourier series representation

$$
d_{111} \psi_{\mathrm{sfe}}=c_{0}+c_{1} f_{1}+c_{2} f_{2}+c_{3} f_{3}+c_{4} f_{4}+a_{1} g_{1}+a_{3} g_{3}
$$

for $\psi_{\text {sfe }}\left(\phi_{1}, \phi_{2}, \phi_{3}\right)$ from Schoeck $(2001,2003,2005,2006)$ with $f_{1,2,3,4}\left(\phi_{1}, \phi_{2}, \phi_{3}\right)$ and $g_{1,3}\left(\phi_{1}, \phi_{2}, \phi_{3}\right)$ fit to DFT data to determine $c_{0}, c_{1}, c_{2}, c_{3}, c_{4}, a_{1}, a_{3}$. Such a truncated Fourier series representation 
has been used in many previous works (Shen and Wang, 2003, 2004; Xiang et al., 2008). To document this in more detail here, consider the fit of (13) to the EAM-potential-based atomic data for $\mathrm{Al}$ and $\mathrm{Au}$ just discussed. To this end, 7 points on the atomic $\gamma$-surface are chosen. These points together with their corresponding position and energy values for $\mathrm{Au}$ and $\mathrm{Al}$ are listed in Table 2 and shown in Figure 1 (below).

\begin{tabular}{|c|c|c|c|}
\hline & $\left(\phi_{\overline{1} 10}, \phi_{\overline{1} \overline{1} 2}\right)$ & $\gamma_{\mathrm{Au}}\left(\mathrm{mJ} / \mathrm{m}^{2}\right)$ & $\gamma_{\mathrm{Al}}\left(\mathrm{mJ} / \mathrm{m}^{2}\right)$ \\
\hline$A$ & $(0,0)$ & 0 & 0 \\
\hline$T_{1}$ & $\left(\frac{1}{4}, 0\right)$ & 184.2 & 212.7 \\
\hline$T$ & $\left(\frac{1}{2}, 0\right)$ & 323.3 & 395.1 \\
\hline$G_{1}$ & $\left(\frac{1}{8}, \frac{1}{8}\right)$ & 51.3 & 65.3 \\
\hline$G_{2}$ & $\left(\frac{1}{4}, \frac{1}{4}\right)$ & 77.2 & 144.2 \\
\hline$G_{3}$ & $\left(\frac{3}{8}, \frac{3}{8}\right)$ & 89.4 & 167.1 \\
\hline$G$ & $\left(\frac{1}{2}, \frac{1}{2}\right)$ & 40.6 & 145.4 \\
\hline
\end{tabular}

Table 2: Selected points on the atomic SFE surface $\gamma_{\mathrm{sfe}}\left(\phi_{\overline{1} 10}, \phi_{\overline{1} \overline{1} 2}\right)$ used to fit (13).

Using these, values for the parameters in (13) have been determined for $\mathrm{Al}$ and $\mathrm{Au}$; these are listed in Table 3.

\begin{tabular}{|c|c|c|c|c|c|c|c|}
\hline & $c_{0}$ & $c_{1}$ & $c_{2}$ & $c_{3}$ & $c_{4}$ & $a_{1}$ & $a_{3}$ \\
\hline $\mathrm{Au}$ & 53.48 & 56.42 & -128.74 & 62.95 & -4.03 & 146.29 & -64.11 \\
\hline $\mathrm{Al}$ & 253.87 & -62.75 & -33.91 & 14.18 & -0.98 & -49.54 & -19.82 \\
\hline
\end{tabular}

Table 3: Identified parameter values in (13) for $\mathrm{Al}$ and $\mathrm{Au}$ based on data in Table 2.

Based on these results, $\gamma_{\text {sfe }}$ and $d_{111} \psi_{\text {sfe }}$ are compared in Figure 1 for Au and in Figure 2 for Al. 

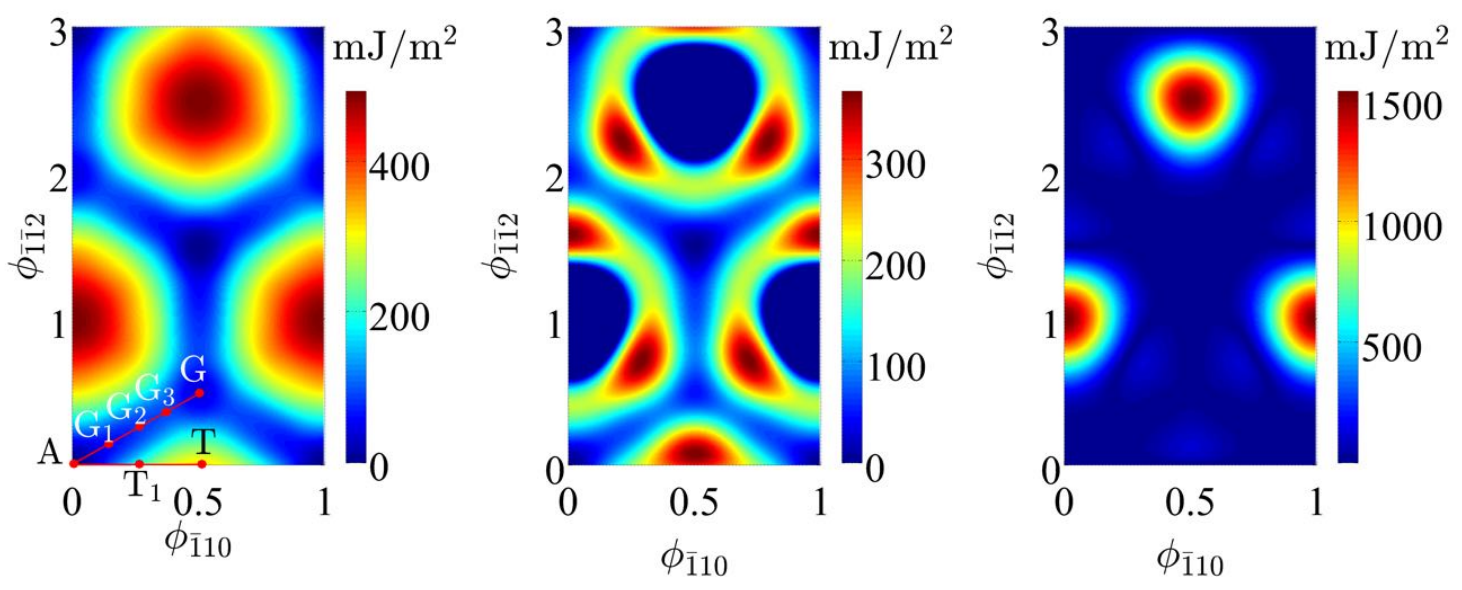

Figure 1: Left: $\gamma_{\text {sfe }}\left(\phi_{\overline{1} 10}, \phi_{\overline{1} \overline{1} 2}\right)$ for Au based on the EAM potential of Grochola et al. (2005). Middle: $d_{111} \psi_{\text {sfe }}$ for Au based on (13) and fit results from Table 3. Right: $\left|\gamma_{\text {sfe }}-d_{111} \psi_{\text {sfe }}\right|$. Note that all negative energy values have been clipped in the contour plots.
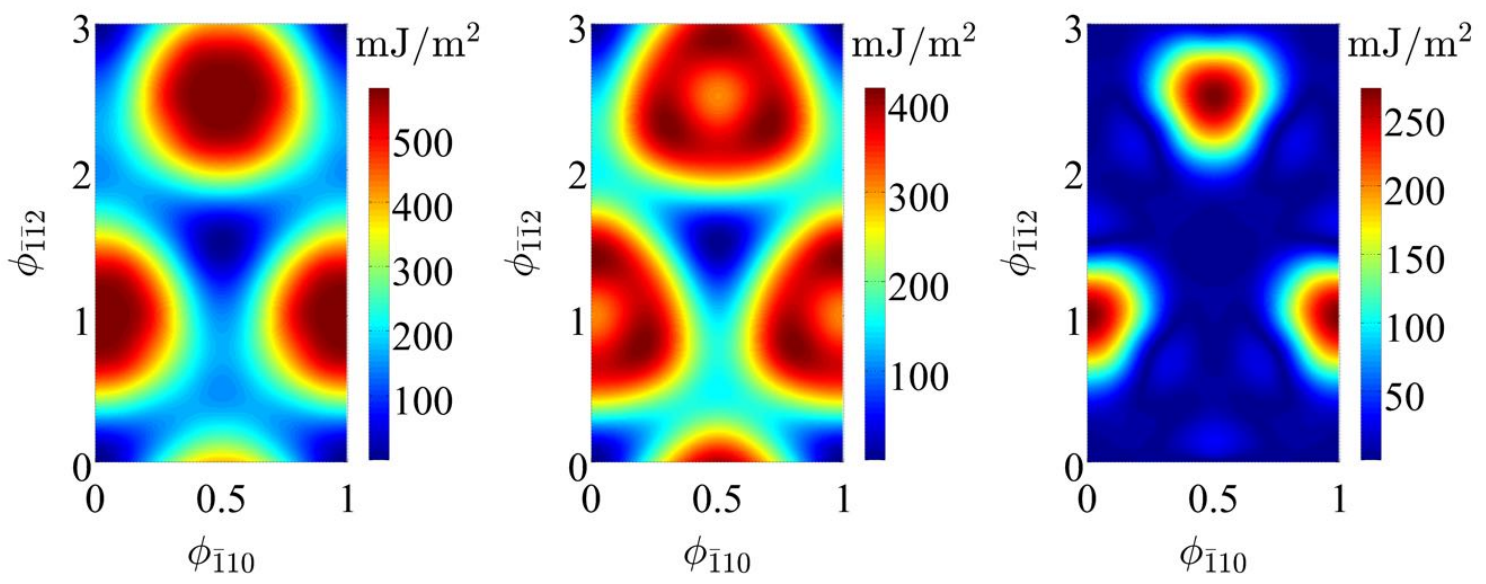

Figure 2: Left: $\gamma_{\mathrm{sfe}}\left(\phi_{\overline{1} 10}, \phi_{\overline{1} \overline{1} 2}\right)$ for Al based on the EAM potential of Mishin et al. (1999). Middle: $d_{111} \psi_{\text {sfe }}$ for Al based on (13) and fit results from Table 3. Right: $\left|\gamma_{\text {sfe }}-d_{111} \psi_{\text {sfe }}\right|$.

As shown in particular by the results for $\left|\gamma_{\text {sfe }}-d_{111} \psi_{\text {sfe }}\right|$ (i.e., Figures 1 and 2 , right), the truncated Fourier series representation is particularly inaccurate in representing the energy peaks. As noted and discussed by Hunter et al. (2011), this is of no consequence for the energy paths in question. For comparability, simulation results from all PF models to follow are based on the direct calculation of $\psi_{\text {sfe }}$ from MS data in look-up table fashion.

\subsection{Gradient energy $\psi_{\mathrm{gra}}$ and energy scaling}

Apart from the determination of $\psi_{\text {ela }}$ and $\psi_{\text {sfe }}$ as discussed above, atomistic data is also used to determine $\psi_{\text {gra }}$ in the model of Mianroodi and Svendsen (2015). As discussed above, this represents the density of energy stored in the transition region between unslipped and slipped regions of the crystal, i.e., in the dislocation line or core. This excludes the SF lying between 
leading and trailing partial dislocations. To determine $\psi_{\text {gra }}$ from atomistic and / or ab initio energy data, Mianroodi and Svendsen (2015) employed the global energy scaling approach of Cahn and Hilliard (1958) adapted to the current context. In particular, this approach couples the scaling $\psi_{\mathrm{H} 0}$ of the extended "homogeneous" (i.e., dependent on $\boldsymbol{\phi}$ ) part $\psi_{\mathrm{H}} \equiv \psi_{\text {ela }}+\psi_{\text {hom }}=\psi_{\text {ela }}+\psi_{\text {sfe }}$ of $\psi$ from (5) and that $\psi_{\mathrm{G} 0}$ of the "gradient" (i.e., dependent on $\nabla \phi$ ) part $\psi_{\mathrm{G}} \equiv \psi_{\text {gra }}$ of $\psi$ from (8) via the relations

$$
\psi_{\mathrm{H} 0}=\frac{\kappa_{\mathrm{G} 0}}{\kappa_{\mathrm{H} 0}} \frac{g_{0}}{l_{0}}, \quad \psi_{\mathrm{G} 0}=\frac{1}{\kappa_{\mathrm{G} 0} \kappa_{\mathrm{H} 0}} g_{0} l_{0} .
$$

In these relations,

$$
g_{0}=\frac{1}{a_{P}} \int_{P} \psi d v
$$

is the energy per unit area in a parallelepiped $P$ of cross sectional area $a_{P}$ perpendicular to the Burgers vector direction of dissociated $\langle 112\rangle$ partials with the MS-based atomic disregistry field $\phi=\sqrt{\phi_{1}^{2}+\phi_{2}^{2}}$. In addition, the length scale

$$
l_{0}=\frac{1}{\sqrt{2}\left|\phi^{\prime}(0)\right|}
$$

is determined by the value $\phi^{\prime}(0)$ of the gradient $\phi^{\prime}$ of $\phi$ in the same direction at the core center. Note that $l_{0}$ represents the length over which the disregistry changes from its non-slipped to its slipped value; in this sense, it is interpreted as a measure of the dislocation core size. Besides $g_{0}$ and $l_{0}, \psi_{\mathrm{H} 0}$ and $\psi_{\mathrm{G} 0}$ in (14) depend on the materially-dependent scaling parameters $\kappa_{\mathrm{H} 0}$ and $\kappa_{\mathrm{G} 0}$ determined in particular by $\psi_{\mathrm{H}} \equiv \psi_{\text {ela }}+\psi_{\text {hom }}=\psi_{\text {ela }}+\psi_{\text {sfe }}$. For more details, the reader is referred to Mianroodi and Svendsen (2015).

Together with those for $g_{0}$ and $l_{0}$, the values of $\kappa_{\mathrm{H} 0}$ and $\kappa_{\mathrm{G} 0}$ for $\mathrm{Al}$ and $\mathrm{Au}$ are given in Table 4.

\begin{tabular}{|c|c|c|c|c|}
\hline & $g_{0} / \mu_{\mathrm{fcc}} d_{111}$ & $l_{0} / d_{111}$ & $\kappa_{\mathrm{H} 0}$ & $\kappa_{\mathrm{G} 0}$ \\
\hline $\mathrm{Au}$ & 0.0547 & 9.62 & 0.1152 & 7.1737 \\
\hline $\mathrm{Al}$ & 0.0626 & 6.17 & 0.1587 & 5.2262 \\
\hline
\end{tabular}

Table 4: Values of the parameters in (14) for $\mathrm{Au}$ and $\mathrm{Al}$ determined from atomistic data as discussed in the text. The effective shear modulus $\mu_{\mathrm{fcc}}=\left(C_{11}-C_{12}+3 C_{44}\right) / 5$ is determined by the data in Table 1 .

In particular, the values for $l_{0}$ in Table 4 are determined for each material from the dissociated edge disregistry field. Again, further details can be found in Mianroodi and Svendsen (2015).

\section{Comparison of simulation results}

Given the two continuum models identified for $\mathrm{Au}$ and $\mathrm{Al}$ as discussed above, we are now in a position to compare their predictions for dislocation processes in these materials. To this end, results for the deformation and disregistry fields of dissociated perfect edge and screw dipoles 
predicted by these two models are compared in detail with each other and with analogous results from MS in what follows.

Since the current PF models are based on the approximations of geometric (i.e., infinitesimal or "small" deformation) and physical (i.e., energy quadratic in linear strain / displacement gradient) linearity, whereas MS-based modeling is inherently non-linear (both geometrically and physically), quantitative agreement of results is not possible. In this context, the degree of discrepancy between the MS and PF modeling results to follow is at least to some degree a measure of the importance of non-linearity for the effect under consideration. Slight additional discrepancies between the quantitative results from the two PF models may also arise due to differences in algorithmic formulation and numerical implementation.

\subsection{Simulation set-up}

As just discussed, the dislocation process under consideration here is dissociation of initially perfect edge and screw dislocations. MS simulation of the edge dipole case is based in particular on a periodic simulation box of orientation

$$
\left(\boldsymbol{i}_{x}, \boldsymbol{i}_{y}, \boldsymbol{i}_{z}\right) \equiv([\overline{1} 10] / \sqrt{2},[111] / \sqrt{3},[11 \overline{2}] / \sqrt{6})
$$

and size of

$$
\left(L_{x}, L_{y}, L_{z}\right)=(120 \sqrt{6}, 180,9 \sqrt{2} / 2) d_{111}
$$

containing $N=259200$ atoms. This is shown in Figure 3 (left).
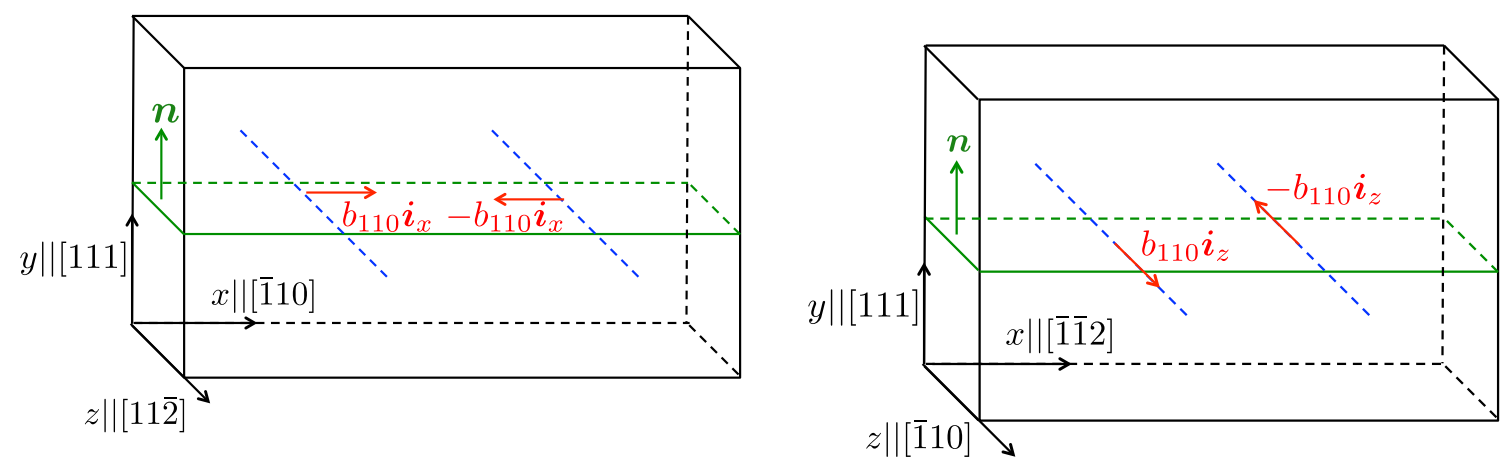

Figure 3: Simulation box set-up for dissociation of an edge dipole (left) and an screw dipole (right).

Analogously,

$$
\left(\boldsymbol{i}_{x}, \boldsymbol{i}_{y}, \boldsymbol{i}_{z}\right) \equiv([\overline{1} \overline{1} 2] / \sqrt{6},[111] / \sqrt{3},[\overline{1} 10] / \sqrt{2})
$$

and

$$
\left(L_{x}, L_{y}, L_{z}\right)=(180 \sqrt{2}, 180,3 \sqrt{6}) d_{111},
$$

are employed in the MS simulation of screw dipole dissociation with the same number of atoms $N=259200$. This is shown in Figure 3 right. 
To initialize the box, the energy is minimized via conjugate gradient relaxation and quadratic line search under constant (zero) stress and constant $(0 \mathrm{~K})$ temperature conditions. In particular, all normal and shear stress components are fixed at zero using the box/relax command in LAMMPS (Plimpton (1995)). The initial dipole configuration in the relaxed box consists of two perfect dislocations with line direction $\boldsymbol{i}_{z}$ and Burgers vector $\pm \boldsymbol{b}\left(b=|\boldsymbol{b}|=a_{0} / \sqrt{2}\right)$ at $(x, y)=\left(L_{x} / 4, L_{y} / 2\right)$ and $\left(3 L_{x} / 4, L_{y} / 2\right)$. These are created by applying the corresponding displacement fields to the atoms as described by Bulatov and Cai (2006, Chapter 5). Dissociation is then simulated via 5000 steps of fast inertial relaxation (using FIRE: Bitzek et al., 2006) followed by 5000 steps of conjugate gradient relaxation, again at zero stress.

For the PF-based simulations of edge dipole dissociation, a structured grid of orientation (17) is employed. The grid spacing is $d_{111}$, and the grid point number is $\left(N_{x}, N_{y}, N_{z}\right)=(294,180,7)$. The resulting grid then consists of $N=N_{x} N_{y} N_{z}=370440$ points. Analogously, in the screw case, a grid is employed with orientation (19), spacing $d_{111}$, point number $\left(N_{x}, N_{y}, N_{z}\right)=(254,180,7)$, and total grid points $N=N_{x} N_{y} N_{z}=320040$ points. Given the symmetry of the dipole configurations in Figure 3, only one half of the simulation box containing one of the two dislocation "poles" (referred to as a monopole) are shown in what follows.

\subsection{Measures of comparison}

Basic kinematic quantities obtained from MS simulations include the disregistry (non-dimensionalized displacement) components $u_{x} / b_{x}$ and $u_{z} / b_{z}$ for dissociated (i.e., partial) dislocations. More specifically, these are obtained from the difference in the atomic displacement of the (111) planes above and below the dislocation core. Recall that $b_{x}=b_{[\overline{1} 10]}$ and $b_{z}=b_{[11 \overline{2}]}$ from (17) in the edge case, and $b_{x}=b_{[\overline{1} \overline{1} 2]}$ and $b_{z}=b_{[\overline{1} 10]}$ from (19) in the screw case. In addition, continuum measures of deformation such as the distortion $\boldsymbol{H}=\nabla \boldsymbol{u}$ and the deformation gradient $\boldsymbol{F}=\boldsymbol{I}+\boldsymbol{H}$ are calculated from the discrete atomic displacement results via the method of Falk and Langer (1998) and Shimizu et al. (2007) as implemented in Ovito (Stukowski, 2010). In what follows, atomic displacement and strain results from MS are compared directly with the corresponding results from the numerical solution of the continuum PF models. The algorithmic determination of these quantities for the model of Mianroodi and Svendsen (2015) as based on (A.1) is presented in more detail in Appendix C. Both MS and PF results for displacement, deformation gradient and strain are visualized in what follows using Ovito (Stukowski, 2010).

As already mentioned above, since the atomic displacement results are non-linear, while the PF models of Hunter et al. (2011, 2013) and Mianroodi and Svendsen (2015) are based on the approximation of (physical and geometric) linearity, the corresponding results cannot agree quantitatively. To look into this in more detail, comparison will also be made with respect to the linear strain $\boldsymbol{E}=\operatorname{sym} \boldsymbol{H}$. In addition, the difference $\boldsymbol{E}_{\mathrm{G}}-\boldsymbol{E}=\frac{1}{2} \boldsymbol{H}^{\mathrm{T}} \boldsymbol{H}$ between the Green strain $\boldsymbol{E}_{\mathrm{G}}$ and $\boldsymbol{E}$ will be examined.

\subsection{Non-linear (i.e., finite) deformation and strain}

Given the different nature of the MS (i.e., non-linear) and PF (i.e., linear) results for deformation, it is reasonable to first examine this issue. To this end, consider for example the case of edge dipole dissociation in Au. In this case, the MS results imply that all components of $\boldsymbol{E}_{\mathrm{G}}-\boldsymbol{E}=\frac{1}{2} \boldsymbol{H}^{\mathrm{T}} \boldsymbol{H}$ 
are of order $10^{-3}$ except the normal component $E_{\mathrm{G} y y}-E_{y y}$ perpendicular to the glide plane, which is of order $10^{-1} . E_{\mathrm{G} y y}$ and $E_{y y}$ are compared in Figure 4.
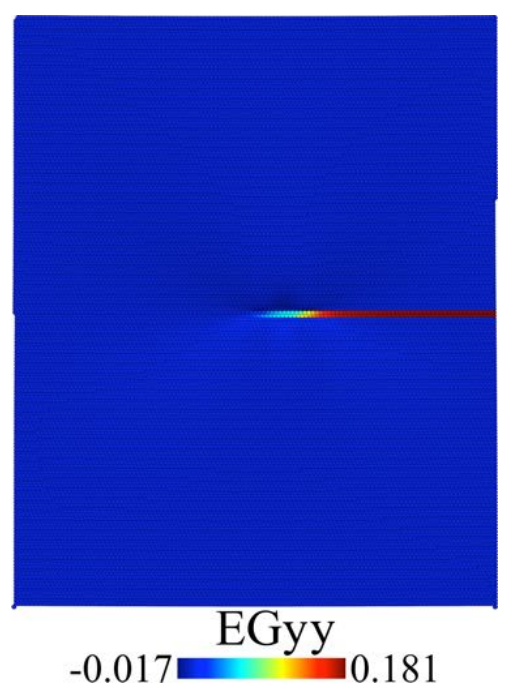

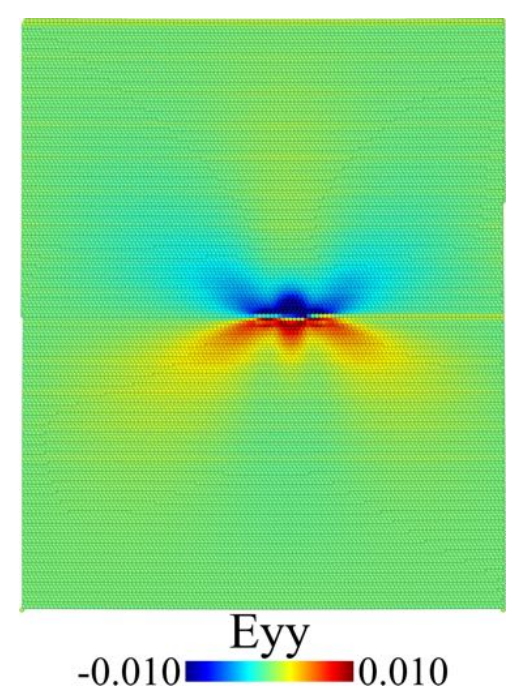

Figure 4: Green $E_{\mathrm{G} y y}$ (left) and linear $E_{y y}$ (right) strain field components of a dissociated edge monopole predicted by MS simulation of edge dipole dissociation (Figure 3, left) in Au. Note that the maximum value of $E_{\text {Gyy }}$ is a factor of 4 smaller than the theoretical (i.e., correct) value. This is due to the fact that the algorithm of Shimizu et al. (2007) used to determine $\boldsymbol{F}$ from atomistic (MS) displacement data breaks down at the core. This is discussed in more detail in the context of Figure 5 below.

To examine this more closely, consider the corresponding results for the displacement and deformation gradient fields in Figure 5.
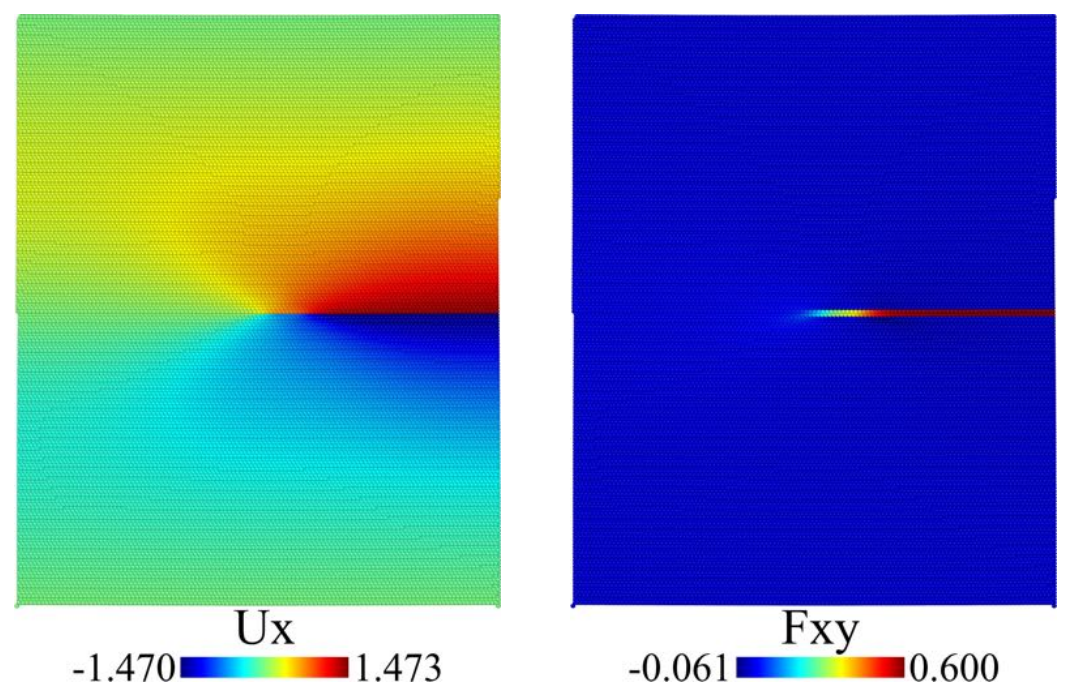

Figure 5: Displacement $u_{x}$ (left) and deformation gradient $F_{x y}$ (right) field components of a dissociated edge monopole predicted by MS simulation of edge dipole dissociation (Figure 3, left) in Au. See text for details.

Note that the maximum value of $F_{x y}$ in Figure 5 is one-half the theoretical value $F_{x y}=b_{110} / d_{111}$ 
for a perfect dislocation. This discrepancy is due to the fact that the method of Shimizu et al. (2007) used to obtain the MS-based results does not work in the core region. In particular, in the current case, this method averages the displacement jump based on one-half of the displaced and non-displaced atoms on the two sides of the glide plane. This results in the factor of two discrepancy. As shown in Figure 5, for an edge dislocation dipole with Burgers vector parallel to $\boldsymbol{i}_{x}, u_{x}$ experiences a jump normal to $\boldsymbol{i}_{y}$, resulting in the relatively large $F_{x y}=H_{x y}$ as seen in Figure 5 (right). Since $F_{x y}$ contributes to $E_{\mathrm{G} y y}=\frac{1}{2}\left(F_{x y}^{2}+F_{y y}^{2}+F_{z y}^{2}-1\right)$, but not to $E_{y y}=F_{y y}-1$, it is clear why $E_{\mathrm{G} y y}$ is orders-of-magnitude larger than $E_{y y}$. In contrast, other components like $E_{\mathrm{G} x y}$ and $E_{x y}$ are relatively close because they both depend on $F_{x y}$. For a dislocation with Burgers vector $\boldsymbol{b}=b \boldsymbol{i}_{x}$ on a glide plane with unit normal $\boldsymbol{n}=\boldsymbol{i}_{y}$, note the linear measure $\operatorname{sym} \boldsymbol{b} \otimes \boldsymbol{n} / d_{111}$ of residual or "plastic" strain has only an $x y$ component. On the other hand, the above MS results imply that slip of such dislocations results in residual strain normal to the slip plane as well. To account for this in the PF-based modeling, the current models need to be generalized to geometric non-linearity, representing work in progress.

\subsection{Displacement and linear strain fields}

For brevity, the model of Hunter et al. $(2011,2013)$ is referred to as the PFDD ("phase-field dislocation dynamics") model, and that of Mianroodi and Svendsen (2015) as the APFM ("atomistic phase-field microelasticity") model, in what follows. As discussed above, Hunter et al. (2011, 2013 ) worked with 3 phase fields per per $\{111\}$ plane, (i.e., one for each $\langle 110\rangle$ direction), whereas Mianroodi and Svendsen (2015) worked with 2 phase fields per $\{111\}$ plane (i.e., one for $\langle 110\rangle$, and one for $\langle 112\rangle)$.

We begin with results for the displacement and strain fields of dissociated edge and screw dipoles in $\mathrm{Al}$ and $\mathrm{Au}$. Results for former case for $\mathrm{Al}$ are shown in Figure 6, and those for $\mathrm{Au}$ in Figure 7. 

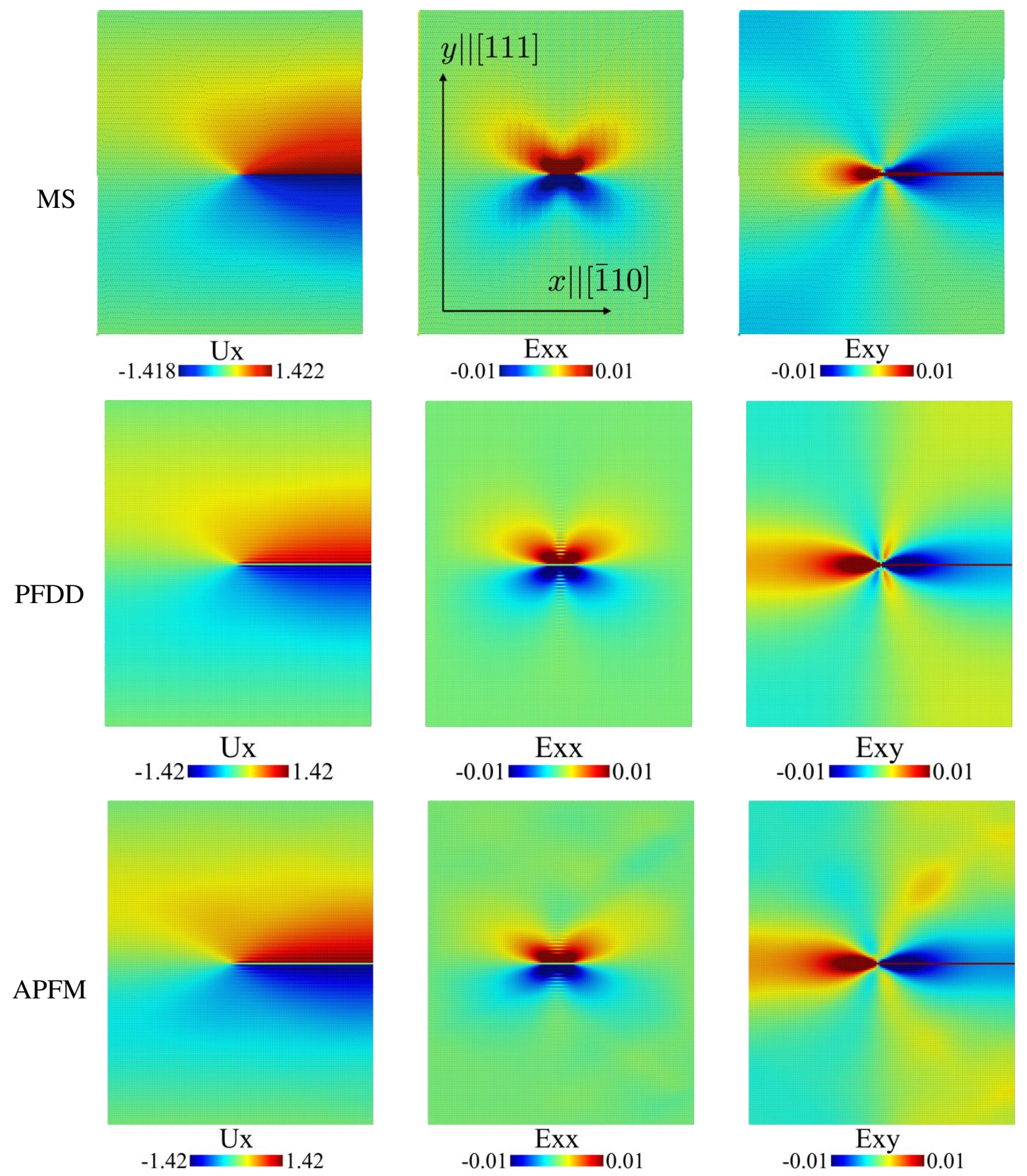

Figure 6: Displacement $u_{x}$ (left), normal strain $E_{x x}$ (middle), and shear strain $E_{x y}$ (right), field components of a dissociated edge monopole in Al predicted by MS (above), PFDD (middle), and APFM (below) in the context of edge dipole dissociation (Figure 3, left). As expected, the deformation fields are largely localized in the core region. In this and all similar figures to follow, note that the contour range is chosen arbitrarily for better visualization. The maximum and minimum field values are actually larger and smaller, respectively, than the values shown in the legends. 

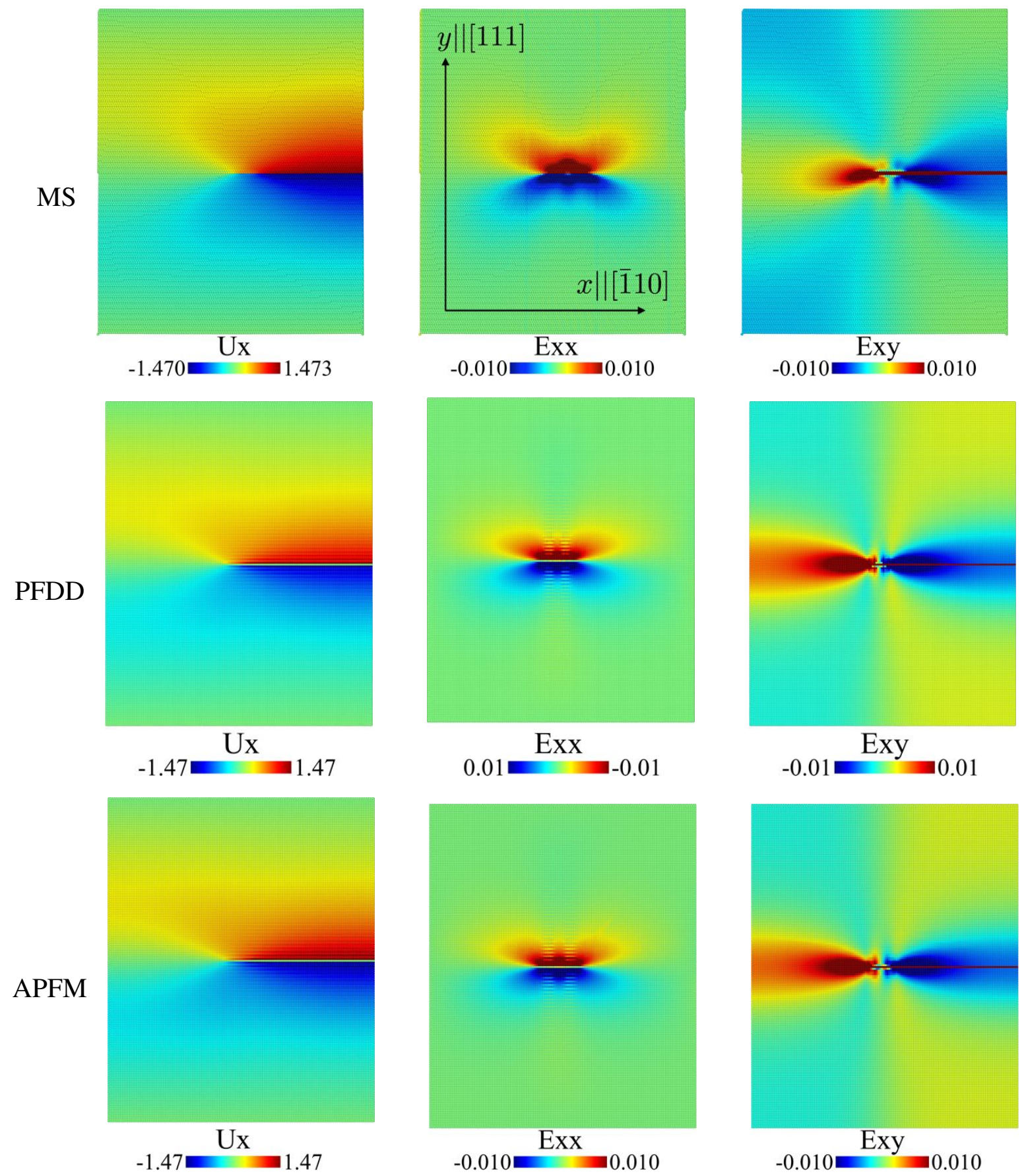

Figure 7: Displacement $u_{x}$ (left), normal strain $E_{x x}$ (middle), and shear strain $E_{x y}$ (right), field components of a dissociated edge monopole in Au predicted by MS (above), PFDD (middle), and APFM (below) in the context of edge dipole dissociation (Figure 3, left).

Analogous results for the dissociated screw dipole case are shown in Figure 8 for $\mathrm{Al}$ and in Figure 9 for $\mathrm{Au}$. 

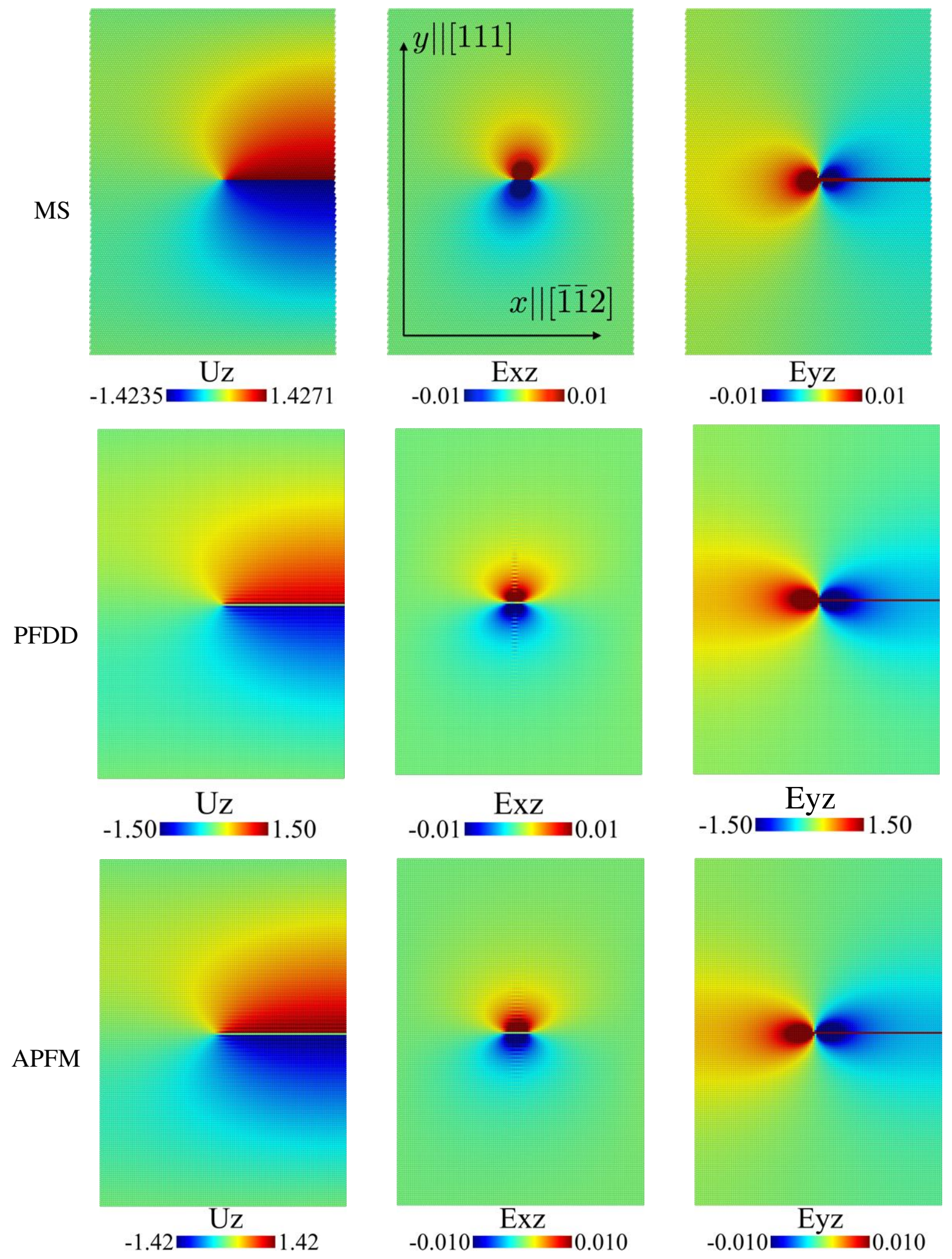

Figure 8: Displacement $u_{z}$ (left), shear strain $E_{x z}$ (middle), and shear strain $E_{y z}$ (right), field components for a dissociated screw dipole in Al predicted by MS (above), PFDD (1 piddle), and APFM (below) in the context of screw dipole dissociation (Figure 3, left). 

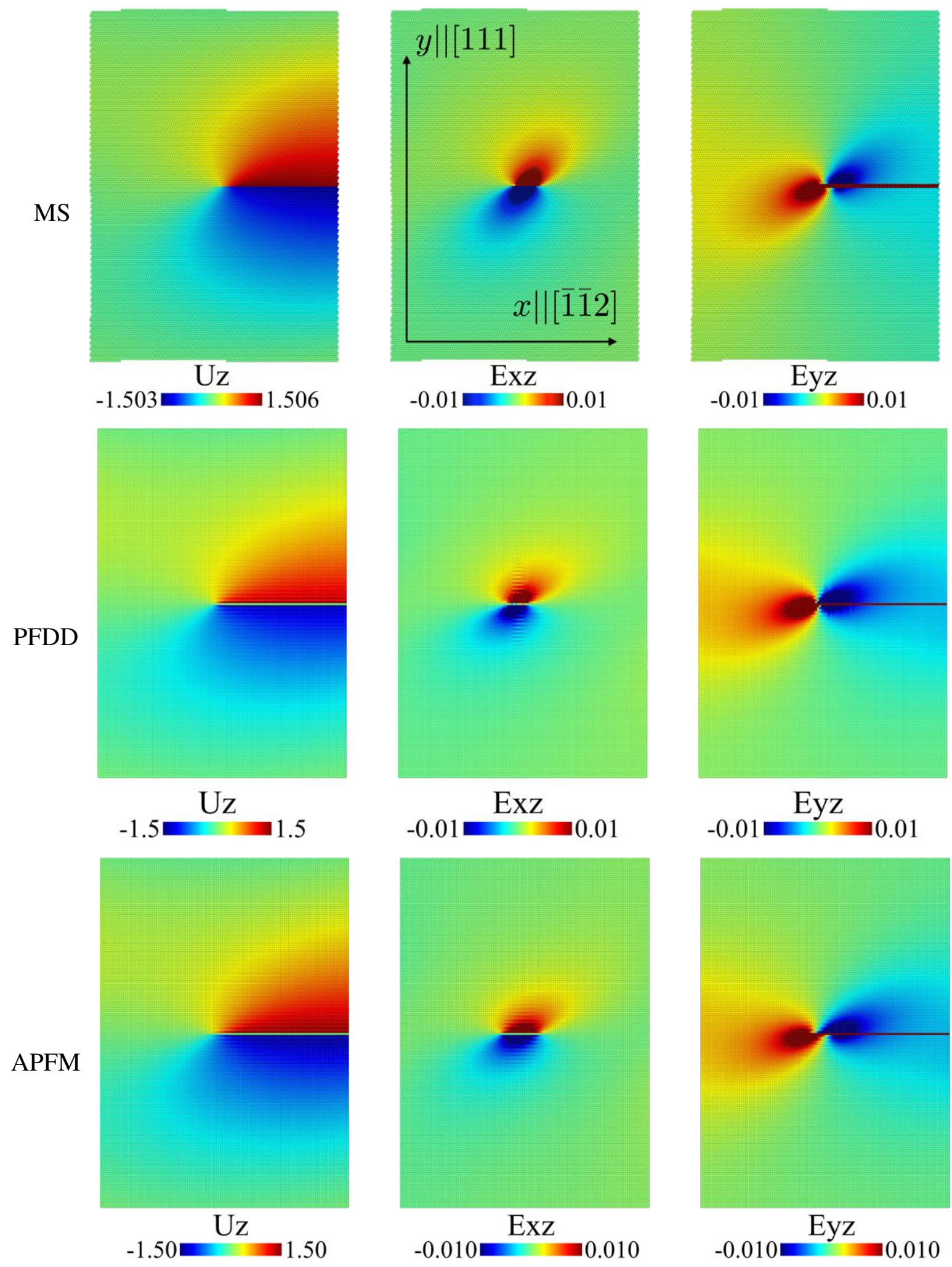

Figure 9: Displacement $u_{z}$ (left), shear strain $E_{x z}$ (middle), and shear strain $E_{y z}$ (right), field components of a dissociated screw monopole in Au predicted by MS (above), PFDD (middle), and APFM (below) in the context of screw dipole dissociation (Figure 3, right). 
Although general agreement between MS, PFDD and APFM results is apparently quite good, differences exist of course in detail. In comparison to the MS and PFDD results, for example, note that the APFM-based results for the $E_{x x}$ and $E_{x y}$ components of the strain field are slightly "flatter", i.e., more two-dimensional or localized to the corresponding glide plane. This is to be expected from the fact that only the projection of $\nabla \phi$ onto the glide plane contributes to $\psi_{\text {gra }}$ in (8). As well, note that any disregistry-based energy storage in the [111] direction has been neglected in both the PFDD and APFM models, something important in other materials than those considered here (e.g., Si: Xiang et al., 2008).

With respect to the results in Figure 6 for the case of edge dissociation in Al, note that the PFDD results for the $E_{x x}$ and $E_{x y}$ components of the strain field are qualitatively similar to those for an perfect Volterra edge dislocation (e.g., Hirth and Lothe, 1982, §3.4). Likewise, the PFDD results for these components of the strain field are qualitatively similar to those for an perfect PN edge dislocation (e.g., Hirth and Lothe, 1982, §8.2). In both cases, this is due to the fact that the dissociated edge dislocation in $\mathrm{Al}$ is almost perfect. Indeed, because of the relatively high SFE of $\mathrm{Al}$ (Section 3.2), the corresponding SF is quite "narrow". In contrast to this, the lower SFE of Au results in significantly wider SFs and corresponding deviation of the strain field components in Figure 7 from their perfect counterparts.

Lastly, consider the above results for the case of screw dissociation in $\mathrm{Al}$ (Figure 8) and $\mathrm{Au}$ (Figure 9). In contrast to the edge case, notable here are the asymmetric displacement and strain fields. The degree of asymmetry is apparently related to the strength $A=2 C_{44} /\left(C_{11}-C_{12}\right)$ of (cubic) elastic anisotropy. In particular, we have $A=1.21$ for $\mathrm{Al}$, and $A=2.9$ for $\mathrm{Au}$. This is confirmed by simulation results assuming elastic isotropy, which predict symmetric core displacement and stress fields for both materials.

\subsection{Disregistry fields}

Consider next a comparison of results for the disregistry fields of dissociated edge and screw dislocations in $\mathrm{Au}$ and $\mathrm{Al}$. To facilitate this, consider the schematic representation of edge (left) and screw (right) dissociation in Figure 10. As shown, in the edge case (left), $\phi_{1} \equiv \phi_{110}=u_{x} / b_{110}$ varies between 0 and 1 , and $\phi_{2} \equiv \phi_{112}=u_{z} / b_{112}$ between 0 and $\frac{1}{2}$. Analogously, in the screw case (right), $\phi_{1} \equiv \phi_{110}=u_{z} / b_{110}$ varies between 0 and 1 , and $\phi_{2} \equiv \phi_{112}=u_{x} / b_{112}$ between 0 and $\frac{1}{2}$.

With the interpretation in Figure 10 in mind, consider first the simulation results for $\mathrm{Au}$ in Figures 11 and 12. 

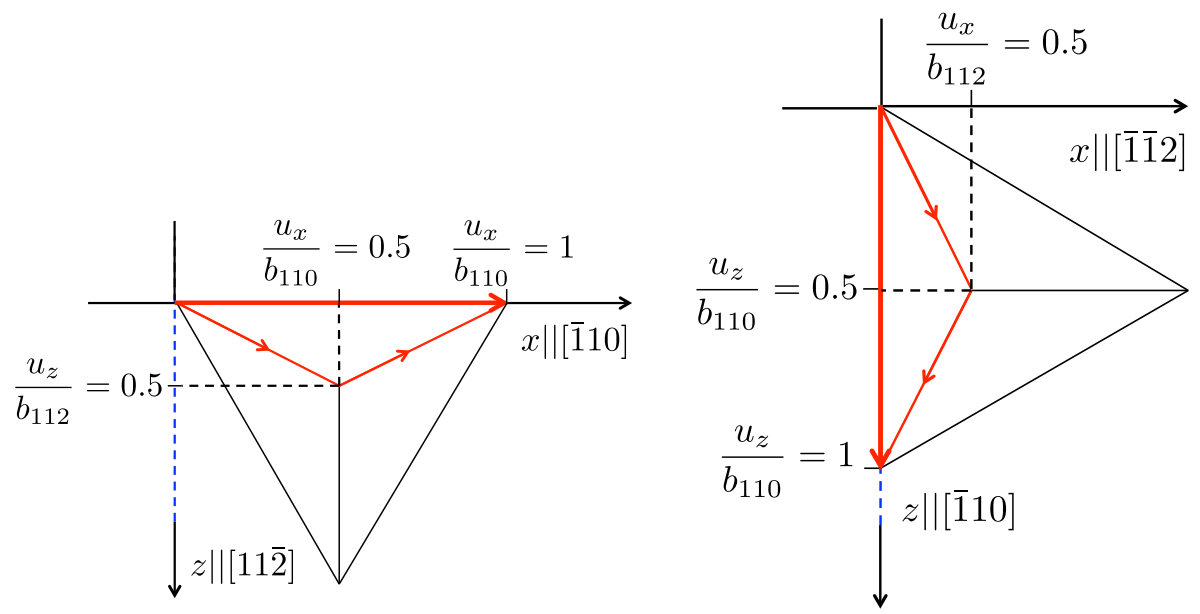

Figure 10: Disregistry components related to the dissociation of an edge (left) and a screw (right) dislocation. See text for details.
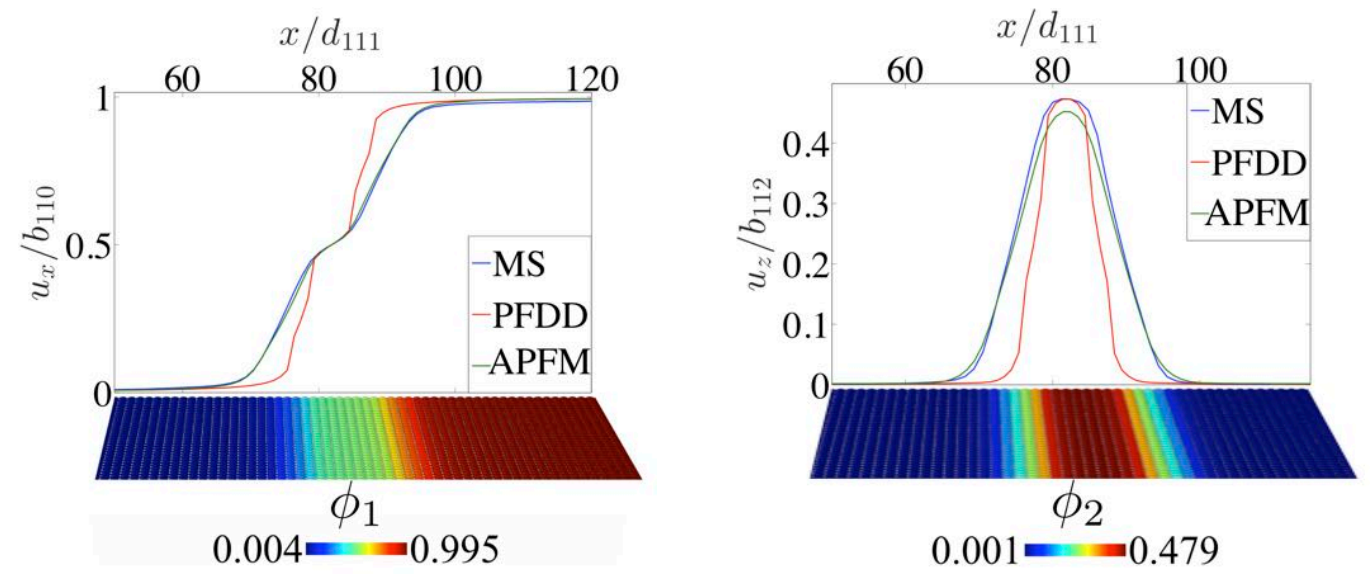

Figure 11: Glide-plane components $\phi_{1}=u_{x} / b_{110}$ (left) and $\phi_{2}=u_{z} / b_{112}$ (right) of the disregistry field of a dissociated edge monopole in Au predicted by MS (blue), PFDD (red) and APFM (green). See Figure 10 for interpretation.
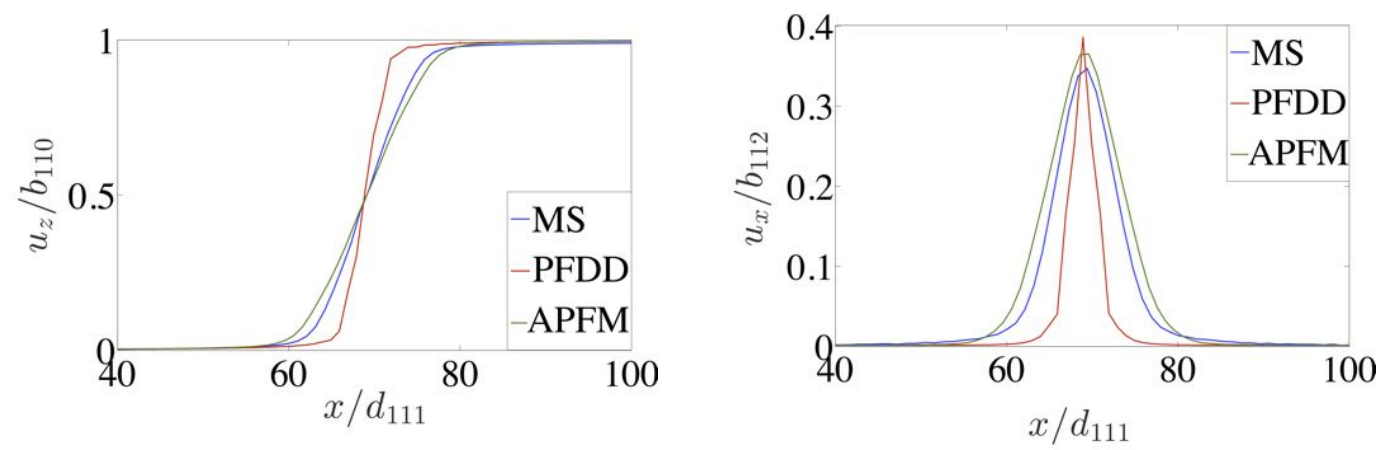

Figure 12: Glide-plane components $\phi_{1}=u_{z} / b_{110}$ (left) and $\phi_{2}=u_{x} / b_{112}$ (right) of the disregistry field of a dissociated screw monopole in Au predicted MS (blue), PFDD (red) and APFM (green). See Figure 10 for interpretation. 
As discussed in Sections 2 and 3.3 above, recall that, in comparison to the PFDD model, the APFM model is based on (i) the additional "gradient" contribution $\psi_{\text {gra }}$ to the free energy density $\psi$ in (5) (Section 2), and (ii) MS-based energy scaling (Section 3.3). Both of these differences are related to the fact that the APFM results (green curves) match the form of the MS-based disregistry profiles (blue curves) more accurately that the PFDD results (red curves). On the other hand, the PFDD results (red curves) in Figures 11 and 12 appear to match the peak values of the MS-based profiles more accurately. This is also in essence the case for $\mathrm{Al}$, as shown by the results in Figures 13 and 14 for a dissociated edge and screw monopole, respectively.
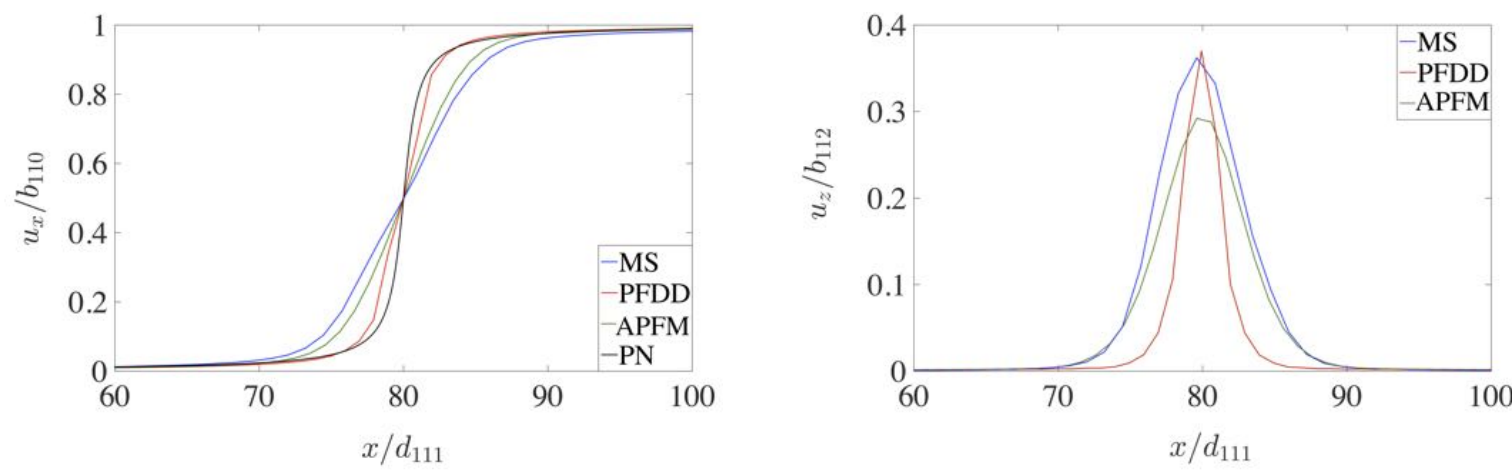

Figure 13: Glide-plane components $\phi_{1}=u_{x} / b_{110}$ (left) $\phi_{2}=u_{z} / b_{112}$ (right) of the disregistry field of a dissociated edge monopole in Al predicted by MS (blue), PFDD (red) and APFM (green). For comparison, the analytical result for $\phi_{1}=u_{x} / b_{110}$ (left, PN, black) from the classic Peierls-Nabarro (PN) model (e.g., Hirth and Lothe, 1982, $\S 8.2$ ) is also shown. See text for details.
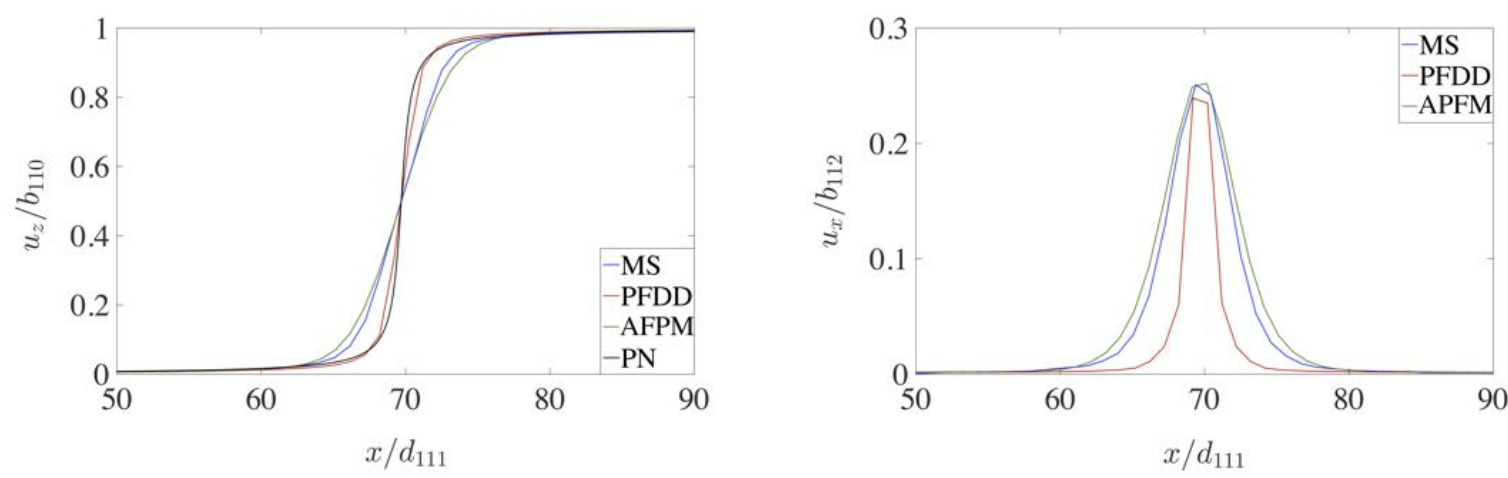

Figure 14: Glide-plane components $\phi_{1}=u_{z} / b_{110}$ (left) and $\phi_{2}=u_{x} / b_{112}$ (right) of the disregistry field of a dissociated screw monopole in Al predicted MS (blue), PFDD (red) and APFM (green). For comparison, the analytical result for $\phi_{1}=u_{z} / b_{110}$ (left, PN, black) from the classic Peierls-Nabarro (PN) model (e.g., Hirth and Lothe, 1982, §8.2) is also shown. See text for details.

One reason for these differences in the PFDD- and APFM-based results for the disregistry profiles is almost certainly the difference in character of the Ginzburg-Landau (GL) relation (12) in the two cases. In particular, local-density-based models like GPN and PFDD result in a HamiltonJacobi-like hyperbolic relation, whereas local-gradient-based PF models like APFM lead to a non- 
linear parabolic relation. Indeed, the former are well-known to result in sharper, and the latter in smoother, profiles, something also evident in the above disregistry results.

As already remarked on above in regards to the results for the shear strain field $E_{x y}$ in Figure 6, the high SFE of $\mathrm{Al}$ (Section 3.2) results in almost no dissociation (i.e., a narrow SF) and an almost perfect dislocation state. This combined with the weak anisotropy of Al motivate the comparison in Figures 13 (left) and 14 (left) with the corresponding analytical results (PN, black) from the classic Peierls-Nabarro (PN) dislocation model (e.g., Hirth and Lothe, 1982, §8.2). Like the PFDD model, the classic PN model neglects in particular $\psi_{\text {gra }}$, which is most likely the main reason for the close agreement of the two in Figures 13 (left) and 14 (left).

More generally, the fact that Al has a relatively high SFE and a narrow SF makes it particularly challenging for PF-based methods to resolve the corresponding MS-based disregistry field. Indeed, in comparison to the case of Au just discussed, which has a lower SFE, the agreement between the MS- and APFM-based results for Al in the edge case in Figure 13 is poorer. As in the case of $\mathrm{Au}$, the peak values of the MS-based disregistry fields are captured better by the PFDD model. Again, this is particularly true for the edge case in Figure 13.

\subsection{Core size \& stacking-fault width}

In this section, disregistry results from the PF models and MS are employed to calculate dislocation core sizes and equilibrium stacking fault widths (SFWs). In particular, analogous to (16), the core size is calculated via

$$
l^{\alpha}=\frac{\left|\phi_{\alpha}(+\infty)-\phi_{\alpha}(-\infty)\right|}{\left|\phi_{\alpha}^{\prime}(0)\right|}
$$

for $\alpha=1,2$ and $\alpha=$ pr, with $\phi_{\mathrm{pr}}:=\sqrt{\phi_{1}^{2}+\phi_{2}^{2}}$ as discussed above (Figure 10). These measures are depicted schematically for the edge case in Figure 15. 

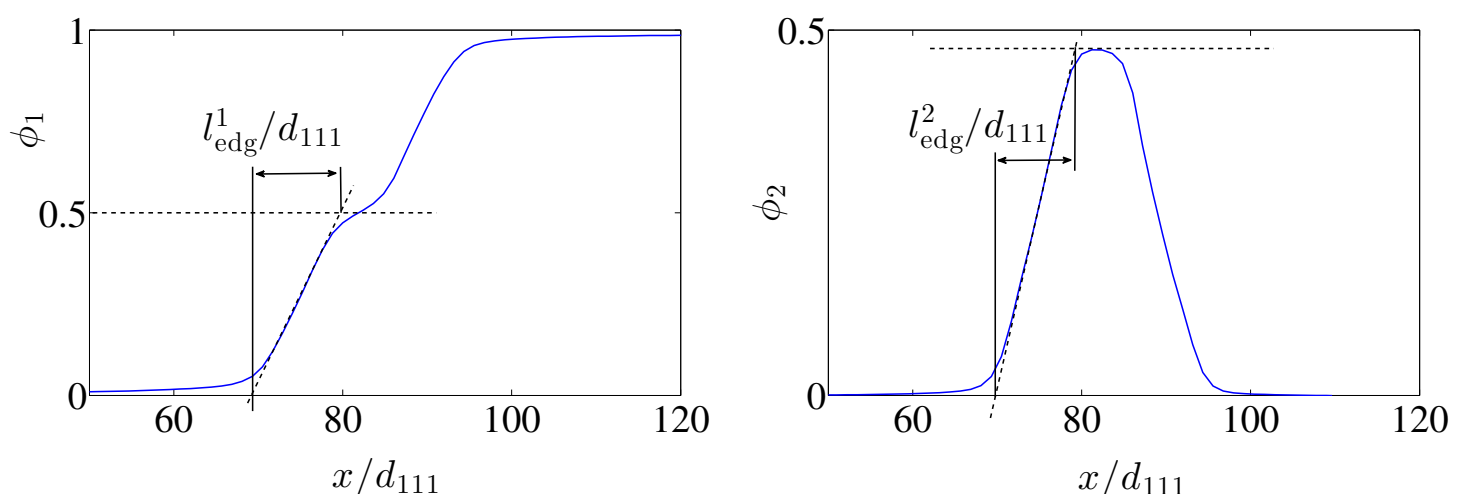

$x / d_{11}$

$x / d_{111}$

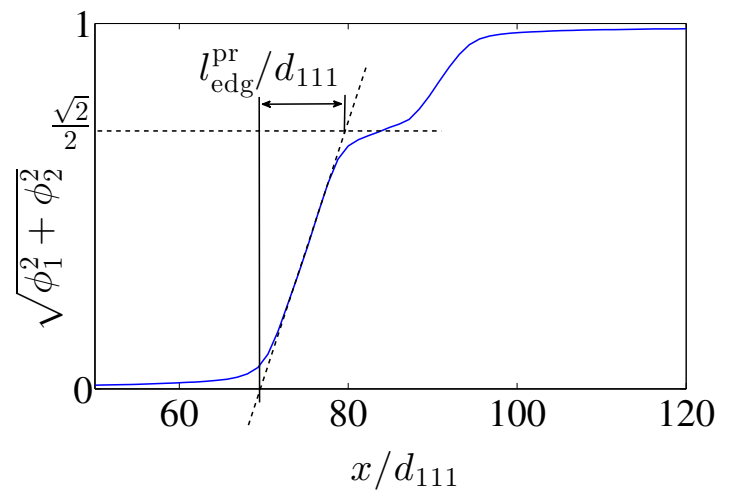

Figure 15: Measures $l_{\text {edg }}^{\alpha}$ of core size from results (here MS) for a dissociated edge dislocation (here Au). See text for details.

Results for $\mathrm{Au}$ and $\mathrm{Al}$ are given in Table 5.

\begin{tabular}{|l||c|c|c||c|c|c|}
\hline \multicolumn{1}{|c||}{} & \multicolumn{3}{c||}{ Au } & \multicolumn{3}{c|}{ Al } \\
\hline & MS & PFDD & APFM & MS & PFDD & APFM \\
\hline$l_{\text {edg }}^{1} / d_{111}$ & 9.7 & 3.5 & 10.1 & 5.7 & 2.6 & 4.6 \\
\hline$l_{\text {edg }}^{2} / d_{111}$ & 8.2 & 3.4 & 9.7 & 4.3 & 2.2 & 4.9 \\
\hline$l_{\text {edg }}^{\mathrm{pr}} / d_{111}$ & 9.6 & 3.6 & 10.4 & 6.2 & 2.9 & 6.4 \\
\hline \hline$l_{\text {scr }}^{1} / d_{111}$ & 5.2 & 4.7 & 7.2 & 3.2 & 1.5 & 3.6 \\
\hline$l_{\text {scr }}^{2} / d_{111}$ & 6.0 & 2.9 & 7.6 & 3.5 & 1.3 & 4.2 \\
\hline$l_{\text {scr }}^{\mathrm{pr}} / d_{111}$ & 7.1 & 2.8 & 8.7 & 4.7 & 2.4 & 5.4 \\
\hline
\end{tabular}

Table 5: Core size measures from (21) for Au and Al. The numerical resolution of all PF results is \pm 0.5 (scaled by $\left.d_{111}\right)$.

As to be expected from the disregistry profile results discussed in Section 4.5, since the slope of APFM- and MS-based disregistry profiles agree better, so do the corresponding core size results in Table 5. 
Next we turn to the equilibrium SFW $d$ as based on phase field and disregistry results. As usual, the SFW represents the dynamic equilibrium between (i) the elastic-energy-based repulsive force between the partials, and (ii) the SFE-based attractive force of the SF on the partials. To calculate $d$, the method of Shen and Wang (2004, Figures 3-5) is employed; this is depicted in Figure 16.
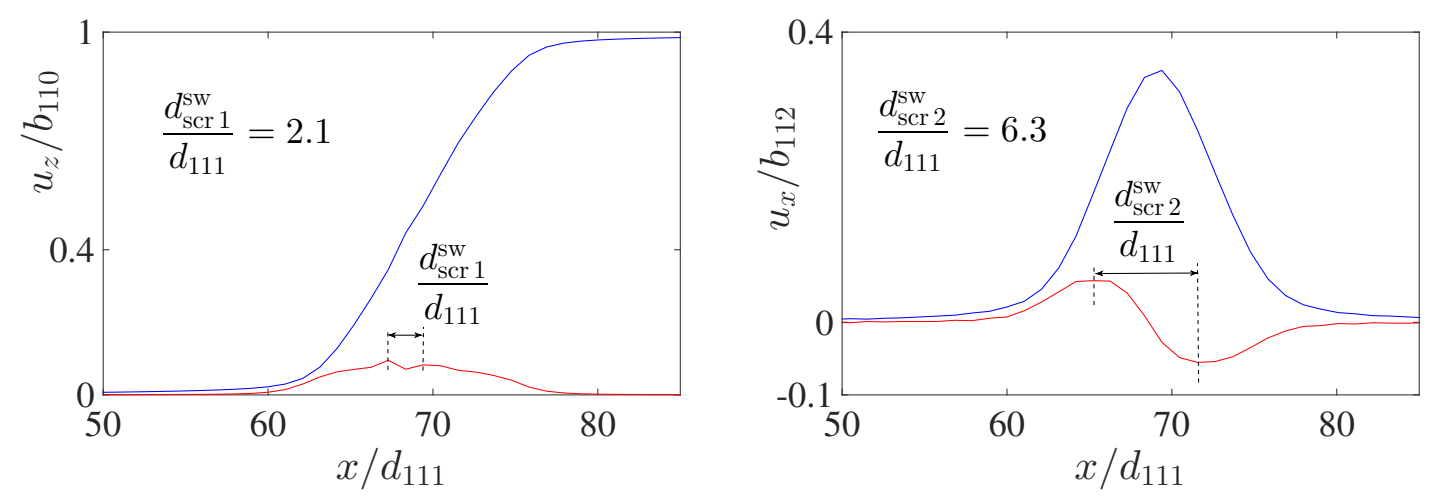

Figure 16: Equlibrium SFWs $d_{\mathrm{scr} 1}^{\mathrm{sw}}$ (left) and $d_{\mathrm{scr} 2}^{\mathrm{sw}}$ (right) derived from $\phi_{1}$ and $\phi_{2}$, respectively, for a dissociated screw monopole (here $\mathrm{Au}$ ). In particular, $d_{\mathrm{scr} 1}^{\mathrm{sw}}$ was used by Shen and Wang (2004). The blue curve is the disregistry profile, and the red curve its gradient. See text for details.

In what follows, corresponding results for $d$ are designated by "sw", i.e., $d^{\text {sw }}$. As depicted in this figure, the distance between the inflection points of $\phi_{1,2}(x)$ determine $d_{1,2}^{\text {sw }}$ from disregistry results. The respective forms of $\phi_{1}(x)$ (left) and $\phi_{2}(x)$ (right) in Figure 16 imply that the inflection points of the former are generally much less well-resolved by a set of discrete disregistry results (either atomic or numerical) than those of the latter. This state of affairs is reflected in the SFW results for $\mathrm{Au}$ and $\mathrm{Al}$ given in Table 6.

\begin{tabular}{|l||c|c|c||c|c|c|}
\hline \multicolumn{1}{|c||}{} & \multicolumn{3}{c||}{ Au } & \multicolumn{3}{c|}{ Al } \\
\hline & MS & PFDD & APFM & MS & PFDD & APFM \\
\hline$d_{\text {edg1 }}^{\mathrm{sw}} / d_{111}$ & 11.9 & 8.6 & 10.0 & 3.6 & 2.5 & - \\
\hline$d_{\mathrm{edg} 2}^{\mathrm{sw}} / d_{111}$ & 10.8 & 8.6 & 10.0 & 6.5 & 2.4 & 6.0 \\
\hline \hline$d_{\mathrm{srr}}^{\mathrm{sw}} / d_{111}$ & 2.1 & 2.4 & 4.0 & 2.1 & - & - \\
\hline$d_{\mathrm{scr2}}^{\mathrm{sw}} / d_{111}$ & 6.3 & 2.4 & 6.0 & 4.6 & 1.2 & 4.0 \\
\hline
\end{tabular}

Table 6: Equilibrium SFW results for dissociated edge and screw dislocations in Au and Al. The numerical resolution of all PF results is \pm 0.5 .

As shown, the PF-based results for $d_{\mathrm{scr} 1}^{\mathrm{sw}}$ are either "significantly" different from those based on MS results or not resolvable with the numerical resolution of the simulations. Again, in general, $d_{1}^{\text {sw }}$ is much more difficult to resolve than $d_{2}^{\text {sw }}$ for a given discrete data set. This is also attested to by the better agreement between the MS and PF (and in particular APFM) results for the latter. In the current case, a much finer numerical discretization than currently possible is required to resolve $d_{1}^{\mathrm{sw}}$ more accurately (this represents work in progress). 
In general, the relative agreement between MS and PF results for $d$ is an indication of the accuracy of $\psi_{\text {ela }}$ and $\psi_{\text {sfe }}$ as approximations to the corresponding atomic quantities. This is consistent with the findings of Shen and Wang (2004), who showed in their parameter study of $\psi_{\mathrm{G} 0}$ that $\psi_{\text {gra }}$ from (8) has a negligible effect on $d$. This is also expected from the point of view that the disregistry field is spatially uniform in the SF and strongly non-uniform in the dislocation core.

\subsection{Dislocation stress field}

Consider finally MS and PF results for the stress field of dissociated dipoles. In particular, the former is based on the determination of atomic volume. To this end, Voronoi tessellation (VT) is employed. Results for this volume and corresponding virial stress results for a dissociated edge dipole in Au are shown in Figure 17.

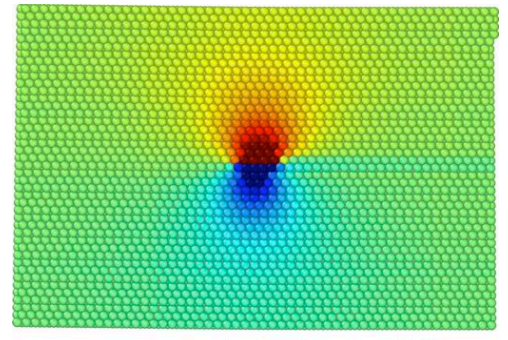

atomic volume $\left(\AA^{3}\right)$

$16.5=17.2$

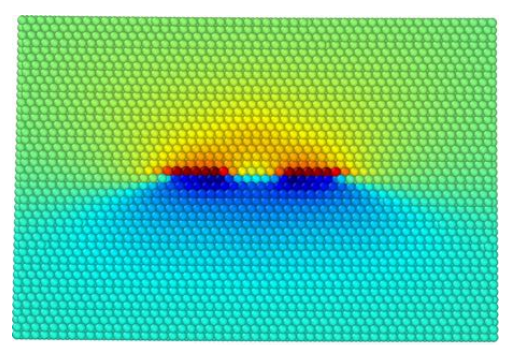

Txx

$-0.115=0.135$

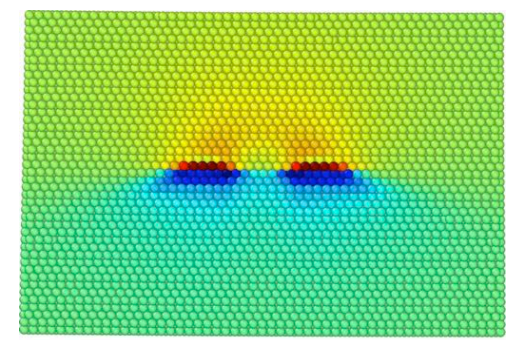

atomic volume $\left(\AA^{3}\right)$ 16.5 17.2

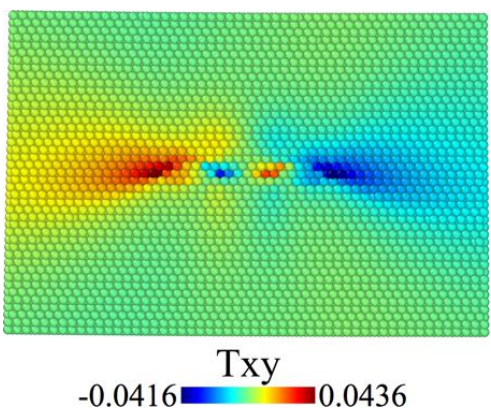

Figure 17: Top: distribution of VT-based atomic volume before (left) and after (right) dissociation of an edge dislocation in Au. Bottom: normal $T_{x x}$ (left) and shear $T_{x y}$ (right) virial stress components scaled by $\mu_{\text {fcc }}=\left(C_{11}-C_{12}+3 C_{44}\right) / 5$ (Burgers vector direction $\boldsymbol{i}_{x}$, glide-plane normal $\boldsymbol{i}_{y}$; see Figure 3 ). For comparison with the VT-based atomic volume results (top), note that the volume of the simulation box divided by the number of atoms is about $16.86 \AA^{3}$; the "nearest-neighbor volume" of $\mathrm{Au}$ is $24.0124 \AA^{3}$ (i.e., $a_{0}^{3} / \sqrt{8}$ ).

The (static) virial stress $\boldsymbol{T}_{i}$ for atom $i$ is given by $\boldsymbol{T}_{i}=\sum_{j \in N_{i}} \boldsymbol{f}_{i j} \otimes \boldsymbol{r}_{i j} / 2 V_{i}$. Here, $N_{i}$ represents the number of atoms neighboring $i, V_{i}$ is the (Voronoi-based) volume of $i, f_{i j}$ is the interaction force between $i$ and $j$ separated by $\boldsymbol{r}_{i j}$. The corresponding PF-based stress field components are shown in Figure 18. 

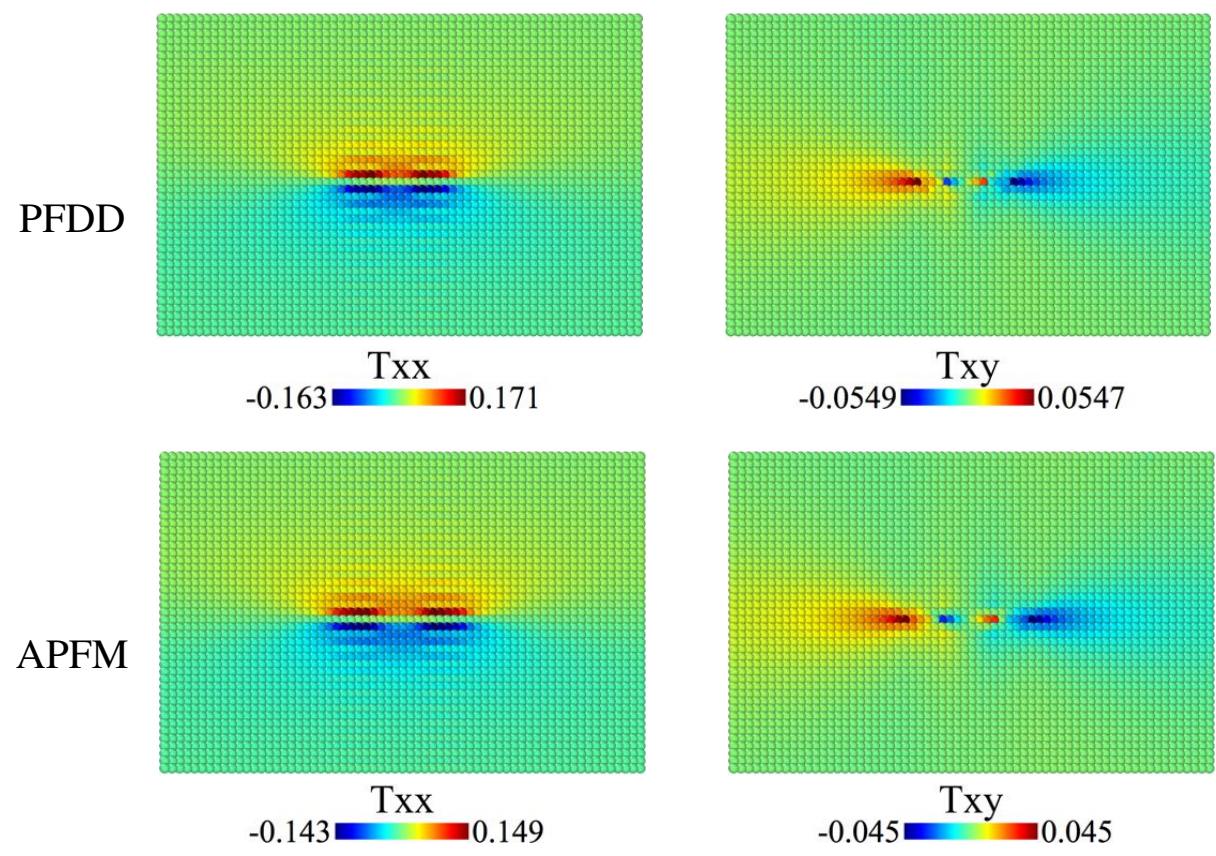

Figure 18: PF-based stress components $T_{x x}$ (left) and $T_{x y}$ (right) from (11) scaled by $\mu_{\text {fcc }}$ for a dissociated edge dipole in Au. These can be compared with the corresponding strain field components in Figure 7.

Comparing the PF results, we note in particular that the magnitude of the PFDD-based dislocation stress field is generally larger than its APFM-based counterpart. This is to be expected from the fact that PFDD predicts smaller core sizes (Table 5) and sharper disregistry profiles (Figure 11) than APFM. Given the qualitative differences between MS-virial-based (Figure 17, bottom) and PF-based (Figure 18, bottom) stress field modeling, (e.g., geometric and physically non-linear versus linear), it is perhaps surprising that the magnitudes of the respective components are rather "close" to each other. In any case, general aspects of the corresponding fields are in qualitative agreement. Further PF-based continuum model development (e.g., generalization to geometric non-linearity) currently in progress will facilitate closer and more detailed comparison of atomistic and continuum results in the future.

\section{Summary and discussion}

In the current work, phase-field models for dislocation processes in fcc crystals have been compared with each other theoretically and with molecular statics in the context of model predictions for displacement, strain and disregistry fields in $\mathrm{Al}$ and $\mathrm{Au}$. Theoretical differences among the models considered (i.e., Shen and Wang, 2004; Xiang et al., 2008; Hunter et al., 2011, 2013; Shen et al., 2014; Mianroodi and Svendsen, 2015) include model forms for the (lattice-based) elastic $\psi_{\text {ela }}$ and (defect-based) slip $\psi_{\text {sli }}$ parts of the free energy (density) $\psi$. In particular, the model formulations for $\psi_{\text {ela }}$ based on residual strain $\boldsymbol{E}_{\mathrm{R}}$ (e.g., Shen and Wang, 2004; Hunter et al., 2011, 2013; Mianroodi and Svendsen, 2015) or on the dislocation tensor curl $\boldsymbol{H}_{\mathrm{R}}$ (e.g., Xiang et al., 2008; Shen 
et al., 2014) were shown in Appendix A to be physically equivalent. In contrast, the respective model forms for $\psi_{\text {sli }}$ are physically distinct. More specifically, Xiang et al. (2008), Hunter et al. (2011, 2013), and Shen et al. (2014) work with $\psi_{\text {sli }}$ modeled on the generalized stacking fault energy (SFE) $\psi_{\text {sfe }}$, whereas Shen and Wang (2004) and Mianroodi and Svendsen (2015) model $\psi_{\text {sli }}$ on $\psi_{\text {sfe }}$ plus an additional contribution $\psi_{\text {gra }}$ associated with dislocation line / core regions. An analogous extension of the basic PN model and approach have been developed by Wang (2008, 2015) with the help of continuum approximations to lattice dynamics. In the context of density functional theory (e.g., Parr and Yang, 1989; Davis, 1996; Engel and Dreizler, 2011), the GPN model is reminiscent of the so-called "local density approximation" (LDA). As has already been discussed above, the (gradient-based) PF model is analogous to the "local gradient approximation" (LGA) to a functional version of $\psi_{\text {sli }}$.

In the second part of the work, predictions of the PF models for the deformation and disregistry fields of dissociated perfect edge and screw dipoles have been compared in detail with each other and with analogous results from MS for $\mathrm{Al}$ and $\mathrm{Au}$. Except for the (geometrically non-linear) variation of $u_{x}$ and $E_{\mathrm{Gyy}}$ normal to the glide plane (see Figures 4 and 5) not accounted for in the geometrically linear PF models considered here, the qualitative and even quantitative agreement between the atomistic and continuum results for the displacement and in-glide-plane strain fields discussed in Section 4.4 is generally quite good.

Due most likely to the fact that the dissociated edge dislocation in $\mathrm{Al}$ is almost perfect, the PFDD and APFM results in Figure 6 are qualitatively quite similar to those for perfect edge dislocations in the analytic PN model (e.g., Hirth and Lothe, 1982). Also notable is the agreement between the MS and PF results for the case of perfect screw dissociation in Al (Figure 8) and Au (Figure 9). In contrast to the edge case, note that the screw case exhibits "asymmetric" displacement and strain fields. As discussed above, the degree of this asymmetry is apparently related to the anisotropy ratio $A$. Examination of additional fcc materials (for example, like those in Hunter et al., 2013) in this regard represents work in progress.

The more detailed, quantitative comparison facilitated by the disregistry results in Section 4.5 implies that a gradient dependence of $\psi_{\text {sli }}$ such as that in (8) in the APFM model results in a spreadout or "diffusion" of the "sharper" PFDD-based disregistry profile to a form closer to that predicted by MS. This is in agreement with the earlier conclusions of Shen and Wang (2004), who showed that the dominant effect of the gradient dependence of $\psi_{\text {sli }}$ is on the partial dislocation (e.g., core size). To a lesser extent, it influences the stacking fault (e.g., width), which is primarily affected by the homogeneous part of $\psi_{\text {sli }}$ (i.e., stacking fault energy) and elastic interaction as accounted for in $\psi_{\text {ela }}$.

The "jump" of $u_{x}$ normal to $\boldsymbol{i}_{y}$ indicated by the large value of $F_{x y}$ in Figure 5 (right) for the dissociated edge monopole in $\mathrm{Au}$ predicted by $\mathrm{MS}$ in $\mathrm{Au}$ (also true for $\mathrm{Al}$ ) implies a strongly planar core morphology. This is well-idealized by the form (8) for the PF gradient energy $\psi_{\text {gra }}$ based on curl $\boldsymbol{H}_{\mathrm{R}}$. For other fcc materials and other crystal structures (e.g., hcp), this will no longer be the case. For example, covalent materials like cubic silicon exhibit non-planar slip (and so core morphologies). This was accounted for by Xiang et al. (2008) in their GPN model via a dependence of $\boldsymbol{H}_{\mathrm{R}}$ on distortion normal to the glide plane (see Appendix B for more details). Investigation of these and other issues such as geometric non-linearity represents work in progress 
to be reported on in the future.

Acknowledgements. A. Hunter and I. J. Beyerlein gratefully acknowledge support from the Los Alamos National Laboratory (LANL) Laboratory Directed Research and Development (LDRD) Program through project numbers LDRD 20160156ER and LDRD 20140348ER, respectively. J. R. Mianroodi and B. Svendsen gratefully acknowledge the support of the German Science Foundation (DFG) for Subproject A10 in the Collaborative Research Center SFB 761.

\section{References}

Allen, S. M., Cahn, J. W., 1979. A macroscopic theory for antiphase boundary motion and its application to antiphase domain coarsening. Acta Metallurgica 27, 1085-1095.

Balluffi, R. W., 2012. Introduction to Elasticity for Crystal Defects. Cambridge University Press, Cambridge.

Bitzek, E., Gahler, F., Koskinen, P., Moseler, M., Gumbsch, P., 2006. Structural Relaxation Made Simple. Physical Review Letters 97 (October), 170201.

Bracewell, R. N., 2000. The Fourier Transform and Its Application, 3rd Edition. McGraw-Hill.

Brown, C. M., Dreyer, W., Müller, W., 2002. Discrete fourier transforms and their application to stress-strain problems in composite mechanics: a convergence study. Proceedings of the Royal Society of London A 458, 1967-1987.

Bulatov, V. V., Cai, W., 2006. Computer Simulation of Dislocations. Oxford Series on Materials Modelling. Oxford.

Cahn, J. W., Hilliard, J. E., 1958. Free energy of a non-uniform system. I. Interfacial energy. Journal of Chemical Physics 28, 258-267.

Chen, L. Q., Shen, J., 1998. Applications of semi-implicit fourier-spectral method to phase field equations. Computer Physics Communications 108, 147-158.

Davis, H. T., 1996. Statistical Mechanics of Phases, Interfaces, and Thin Films. Advances in Interfacial Engineering Series. Wiley-VCH, New York, Ch. 9.1: Calculus of variations and functional derivatives, pp. 425-435.

Dreyer, W., Müller, W., 2000. A study of the coarsening of tin/lead solders. International Journal of Solids and Structures 37, 3841-3871.

Engel, E., Dreizler, R. M., 2011. Density Functional Theory. Springer.

Eshelby, J. D., 1951. The force on an elastic singularity. Philosophical Transactions of the Royal Society of London A 244, 87-112.

Eshelby, J. D., 1956. Continuum theory of defects. In: Turnbull, D. (Ed.), Solid State Physics. Vol. 3. Academic Press, pp. 79-144.

Falk, M. L., Langer, J. S., 1998. Dynamics of viscoplastic deformation in amorphous solids. Physical Review E 57 (6), 7192-7205.

Grochola, G., Russo, S. P., Snook, I. K., 2005. On fitting a gold embedded atom method potential using the force matching method. The Journal of Chemical Physics 123, 204719.

Hirth, J. P., Lothe, J., 1982. Theory of Dislocations, 2nd Edition. Wiley, New York.

Hunter, A., Beyerlein, I. J., Germann, T. C., Koslowski, M., 2011. Influence of the stacking fault energy surface on partial dislocations in fec metals with a three dimensional phase field dynamics model. Physical Review B 84, 144108.

Hunter, A., Zhang, R. F., Beyerlein, I. J., Germann, T. C., Koslowski, M., 2013. Dependence of equilibrium stacking fault width in fcc metals on the gamma-surface. Modelling and Simulation in Materials Science and Engineering 21, 025015 (19pp).

Iyer, M., Radhakrishnan, B., Gavini, V., 2015. Electronic-structure study of an edge dislocation in aluminum and the role of macroscopic deformations on its energetics. Journal of the Mechanics and Physics of Solids 76, 260-275.

Khachaturyan, A. G., 1983. Theory of Structural Transformations in Solids. Wiley, New York.

Kröner, E., 1959. Allgemeine Theorie der Versetzungen und Eigenspannungen. Archive for Rational Mechanics and Analysis 4 (Supplement 1), 273-334.

Li, S., Wang, G., 2008. Introduction to Micromechanics and Nanomechanics. World Scientific, Singapore. 
Mianroodi, J. R., Svendsen, B., 2015. Atomistically determined phase field modeling of dislocation dissociation, stacking fault formation, dislocation slip, and reactions in fcc systems. Journal of the Mechanics and Physics of Solids 77, 109-122.

Mishin, Y., Farkas, D., Mehl, M. J., Papaconstantopoulos, D. A., 1999. Interatomic potentials for monoatomic metals from experimental data and ab initio calculations. Physical Review B 59, 3393.

Monchiet, V., Bonnet, G., 2012. A polarization-based fft iterative scheme for computing the effective properties of elastic composites with arbitrary contrast. International Journal for Numerical Methods in Engineering 89, 14191436.

Moulinec, H., Suquet, P., 1994. A fast numerical method for computing the linear and nonlinear mechanical properties of composites. Comptes Rendus de l'Académie des Sciences Paris 318, 1417-1423.

Mura, T., 1963. Continuous distribution of moving dislocations. Philosophical Magazine 8, 843-857.

Mura, T., 1987. Micromechanics of Defects in Solids. Martinus Nijhoff, Dordrecht.

Nani, E. S., Gururajan, M. P., 2014. On the incorporation of cubic and hexagonal interfacial energy anisotropy in phase field models using higher order tensor terms. Philosophical Magazine 94, 3331-3352.

Nemat-Nasser, S., Hori, M., 1999. Micromechanics: Overall Properties of Heterogeneous Materials. Elsevier, Amsterdam.

Nye, J., 1953. Some geometric relations in dislocated crystals. Acta Metallurgica 1, 153-162.

Parr, R. G., Yang, W., 1989. Density-Functional Theory of Atoms and Molecules. Oxford University Press, Oxford, Ch. Appendix A, pp. 246-254.

Plimpton, S., 1995. Fast parallel algorithms for short-range molecular dynamics. Journal of Computational Physics 117 (June 1994), 1-42.

Schoeck, G., 2001. The core structure, recombination energy and Peierls energy for dislocations in Al. Philosophical Magazine A 81, 1161.

Schoeck, G., 2003. The emission of dislocations from crack tips - a critical assessment. Material Science and Engineering A 356, 93-101.

Schoeck, G., 2005. The Peierls model: progress and limitations. Material Science and Engineering A 400-401, 7-17.

Schoeck, G., 2006. The core structure of dislocations: Peierls model versus atomic simulation. Acta Materialia 54, 4865-4870.

Shen, C., Ju, L., Wang, Y., 2014. Predicting structure and energy of dislocations and grain boundaries. Acta Materialia 74, 125-131.

Shen, C., Wang, Y., 2003. Phase field modeling of dislocation networks. Acta Materialia 51, 2595-2610.

Shen, C., Wang, Y., 2004. Incorporation of gamma-surface to phase field model of dislocations: simulation dislocation dissociation in fcc crystals. Acta Materialia 52, 683-691.

Shimizu, F., Ogata, S., Li, J., 2007. Theory of shear banding in metallic glasses and molecular dynamics calculations. Materials Transactions 48, 2923-2927.

Stukowski, A., 2010. Visualization and analysis of atomistic simulation data with OVITO-the Open Visualization Tool. Modelling and Simulation in Materials Science and Engineering 18, 015012.

Suquet, P., 1997. Continuum Micromechanics. Vol. 377 of CISM International Center for Mechanical Sciences. Springer, Berlin.

Teodosiu, C., 1982. Elastic Theory of Crystals. Amsterdam. North Holland.

Wang, R., Wang, S., Wu, X., 2011. Edge dislocation core structures in fcc metals determined from ab initio calculations combined with the improved Peierls-Nabarro equation. Physica Scripta 83, 045604(7pp).

Wang, S., 2008. Dislocation equation from the lattice dynamics. Journal Physics A: Mathematical and Theoretical 41, 015005.

Wang, S., 2015. The dislocation equation as a generalization of the Peierls equation. Philosophical Magazine DOI: 10.1080/14786435.2015.1096027.

Wang, Y., Li, J., 2010. Phase field modeling of defects and deformation. Acta Materialia 58, 1212-1235.

Wang, Y. U., Jin, Y. M., Cutiño, A. M., Khachaturyan, A. G., 2001. Nanoscale phase field microelasticity theory of dislocations: model and 3d simulations. Acta Materialia 49, 1847-1857.

Willis, J. R., 1967. Second-order effects of dislocations in anisotropic crystals. International Journal of Engineering Science 5, 171-190. 
Xiang, Y., Wei, H., Ming, P., E, W., 2008. A generalized Peirls-Nabarro model for curved dislocations and core structures of dislocation loops in $\mathrm{Al}$ and $\mathrm{Cu}$. Acta Materialia 56, 1447-1460.

\section{Appendix A. Comparison of models for elastic energy \& stress}

\section{Appendix A.1. Basic formulation}

As discussed in the text, PF models for defective crystals such as those of Shen and Wang (2004), Hunter et al. (2011, 2013), or Mianroodi and Svendsen (2015), are based in particular on periodic (i.e., purely bulk) homogeneous single-crystal linear elastic relations for $\psi_{\text {ela }}$ of the form in (6) depending on $\boldsymbol{H}_{\mathrm{R}}$ (e.g., Eshelby, 1951, 1956; Kröner, 1959; Hirth and Lothe, 1982; Teodosiu, 1982; Khachaturyan, 1983; Mura, 1987; Li and Wang, 2008; Balluffi, 2012). In contrast, those of Xiang et al. (2008) and Shen et al. (2014) are based on generalization of the Peierls-Nabarro model (e.g., Wang and Li, 2010) in which $\psi_{\text {ela }}$ and $\boldsymbol{T}$ are formulated in terms of $\boldsymbol{A}_{\mathrm{R}}=\operatorname{curl} \boldsymbol{H}_{\mathrm{R}}$. In essence, these have been shown to be equivalent by Mura $(1963,1987)$ in the dynamic case, and by Willis (1967, Appendix 1) in the static case Teodosiu (see also 1982, §18.3). For completeness, an independent derivation of this equivalence is provided here.

As usual, this begins with the basic result

$$
\boldsymbol{u}(\boldsymbol{x})=\int \boldsymbol{G}_{\mathrm{E}}\left(\boldsymbol{x}-\boldsymbol{x}^{\prime}\right) \operatorname{div}^{\prime} \boldsymbol{T}_{\mathrm{R}}\left(\boldsymbol{x}^{\prime}\right) d v\left(\boldsymbol{x}^{\prime}\right)=: \boldsymbol{G}_{\mathrm{E}} * \operatorname{div} \boldsymbol{T}_{\mathrm{R}}(\boldsymbol{x})
$$

(e.g., Teodosiu, 1982; Khachaturyan, 1983; Mura, 1987; Suquet, 1997; Li and Wang, 2008) for the displacement field $\boldsymbol{u}$ as a particular solution of (10) with (11) in terms of the residual stress

$$
\boldsymbol{T}_{\mathrm{R}}:=-C_{\mathrm{E}} \boldsymbol{E}_{\mathrm{R}}=-C_{\mathrm{E}} \boldsymbol{H}_{\mathrm{R}}
$$

and linear elastic Green function $\boldsymbol{G}_{\mathrm{E}}$ based on $\boldsymbol{C}_{\mathrm{E}}$ satisfying

$$
\operatorname{div} C_{\mathrm{E}} \operatorname{sym} \nabla\left(\boldsymbol{G}_{\mathrm{E}}\left(\boldsymbol{x}-\boldsymbol{x}^{\prime}\right) \boldsymbol{a}\right)=-\delta\left(\boldsymbol{x}-\boldsymbol{x}^{\prime}\right) \boldsymbol{a}
$$

for any constant vector $\boldsymbol{a}$. As usual, the symmetry of $\boldsymbol{C}_{\mathrm{E}}$ implies $\boldsymbol{G}_{\mathrm{E}}^{\mathrm{T}}=\boldsymbol{G}_{\mathrm{E}}$. As well,

$$
\nabla^{\prime} \boldsymbol{G}_{\mathrm{E}}=-\nabla \boldsymbol{G}_{\mathrm{E}}
$$

holds via the distributional character of $\boldsymbol{G}_{\mathrm{E}}$ (e.g., Teodosiu, 1982; Mura, 1987; Li and Wang, 2008; Balluffi, 2012). To simplify the algebra, let $\boldsymbol{g}_{\boldsymbol{a}}:=\boldsymbol{G}_{\mathrm{E}} \boldsymbol{a}$ for any constant $\boldsymbol{a}$. Using this, $\boldsymbol{a} \cdot \boldsymbol{u}=: \boldsymbol{g}_{\boldsymbol{a}} \star \operatorname{div} \boldsymbol{T}_{\mathrm{R}}$ is obtained from (A.1) for $\boldsymbol{u}$ with respect to an arbitrary vector $\boldsymbol{a}$ via the symmetry of $\boldsymbol{G}_{\mathrm{E}}$. Here and in what follows, the operator $\star$ represents both convolution and a scalar product of tensors. Via (A.2) and the symmetry of $C_{\mathrm{E}}$, partial integration, the divergence theorem, boundedness, and (A.4), yield the form

$$
\boldsymbol{a} \otimes \boldsymbol{c} \cdot \nabla \boldsymbol{u}=\boldsymbol{a} \cdot \nabla_{\boldsymbol{c}} \boldsymbol{u}=-\nabla_{\boldsymbol{c}} \boldsymbol{C}_{\mathrm{E}}\left[\nabla \boldsymbol{g}_{\boldsymbol{a}}\right] \star \boldsymbol{H}_{\mathrm{R}}
$$

for the total equilibrium distortion $\nabla \boldsymbol{u}$ in terms of $\boldsymbol{H}_{\mathrm{R}}$, with $\nabla_{\boldsymbol{c}} \boldsymbol{u}:=(\nabla \boldsymbol{u}) \boldsymbol{c}$. The result (A.5) is the starting point for the following derivation of two relations for the elastic strain $\boldsymbol{E}_{\mathrm{E}}=\operatorname{sym} \boldsymbol{H}_{\mathrm{E}}$ with $\boldsymbol{H}_{\mathrm{E}}=\nabla \boldsymbol{u}-\boldsymbol{H}_{\mathrm{R}}$. Consider first that based on the dislocation tensor

$$
\boldsymbol{A}_{\mathrm{R}}:=\operatorname{curl} \boldsymbol{H}_{\mathrm{R}}=-\operatorname{curl} \boldsymbol{H}_{\mathrm{E}}=-\boldsymbol{A}_{\mathrm{E}}
$$


(e.g., Nye, 1953) with

$$
\left(\operatorname{curl} \boldsymbol{H}_{\mathrm{R}}\right)(\boldsymbol{a} \times \boldsymbol{c}):=\left(\nabla_{\boldsymbol{a}} \boldsymbol{H}_{\mathrm{R}}\right) \boldsymbol{c}-\left(\nabla_{\boldsymbol{c}} \boldsymbol{H}_{\mathrm{R}}\right) \boldsymbol{a} .
$$

To simplify notation, let $\boldsymbol{T}_{\boldsymbol{a}}:=\boldsymbol{C}_{\mathrm{E}}\left[\nabla \boldsymbol{g}_{\boldsymbol{a}}\right]$. Via boundedness, the divergence theorem, and (A.4), (A.5) can be expressed in the alternative form

$$
\boldsymbol{a} \cdot \nabla_{\boldsymbol{c}} \boldsymbol{u}=-\nabla_{\boldsymbol{c}} \boldsymbol{T}_{\boldsymbol{a}} \star \boldsymbol{H}_{\mathrm{R}}=-\boldsymbol{T}_{\boldsymbol{a}} \star \nabla_{\boldsymbol{c}} \boldsymbol{H}_{\mathrm{R}} .
$$

From (A.6) and (A.7) follows

$$
\nabla_{c} \boldsymbol{H}_{\mathrm{R}}=\boldsymbol{A}_{\mathrm{R}} \text { axt } \boldsymbol{c}+\nabla\left(\boldsymbol{H}_{\mathrm{R}} \boldsymbol{c}\right)
$$

with (axt $\boldsymbol{c}) \boldsymbol{a}:=\boldsymbol{c} \times \boldsymbol{a}$. Consequently, (A.8) reduces to

$$
\boldsymbol{a} \cdot \nabla_{\boldsymbol{c}} \boldsymbol{u}=\boldsymbol{T}_{\boldsymbol{a}} \text { axt } \boldsymbol{c} \star \boldsymbol{A}_{\mathrm{R}}-\boldsymbol{T}_{\boldsymbol{a}} \star \nabla\left(\boldsymbol{H}_{\mathrm{R}} \boldsymbol{c}\right),
$$

via the skew-symmetry of axt $c$. Integration by parts, the divergence theorem and boundedness imply

$$
-\boldsymbol{T}_{\boldsymbol{a}} \star \nabla\left(\boldsymbol{H}_{\mathrm{R}} \boldsymbol{c}\right)=\int \operatorname{div}^{\prime} \boldsymbol{T}_{\boldsymbol{a}} \cdot \boldsymbol{H}_{\mathrm{R}}^{\prime} \boldsymbol{c} d v^{\prime}=\delta \boldsymbol{a} \star \boldsymbol{H}_{\mathrm{R}} \boldsymbol{c}=\boldsymbol{a} \otimes \boldsymbol{c} \cdot \boldsymbol{H}_{\mathrm{R}}
$$

since $\operatorname{div}^{\prime} \boldsymbol{T}_{\boldsymbol{a}}=-\operatorname{div} \boldsymbol{T}_{\boldsymbol{a}}=\delta \boldsymbol{a}$ via (A.3) and (A.4) when $\boldsymbol{C}_{\mathrm{E}}$ is constant. Combining (A.10) and (A.11) results then in the alternative form

$$
\boldsymbol{a} \otimes \boldsymbol{c} \cdot \nabla \boldsymbol{u}=C_{\mathrm{E}}\left[\nabla \boldsymbol{g}_{\boldsymbol{a}}\right] \operatorname{axt} \boldsymbol{c} \star \boldsymbol{A}_{\mathrm{R}}+\boldsymbol{a} \otimes \boldsymbol{c} \cdot \boldsymbol{H}_{\mathrm{R}}
$$

of (A.5) in terms of $\boldsymbol{A}_{\mathrm{R}}$. In turn, (A.5) and (A.12) imply

$$
\boldsymbol{a} \otimes \boldsymbol{c} \cdot \boldsymbol{H}_{\mathrm{E}}=-\nabla_{\boldsymbol{c}} \boldsymbol{C}_{\mathrm{E}}\left[\nabla \boldsymbol{g}_{\boldsymbol{a}}\right] \star \boldsymbol{H}_{\mathrm{R}}-\boldsymbol{a} \otimes \boldsymbol{c} \cdot \boldsymbol{H}_{\mathrm{R}}=\boldsymbol{C}_{\mathrm{E}}\left[\nabla \boldsymbol{g}_{\boldsymbol{a}}\right] \text { axt } \boldsymbol{c} \star \boldsymbol{A}_{\mathrm{R}}
$$

for $\boldsymbol{H}_{\mathrm{E}}=\nabla \boldsymbol{u}-\boldsymbol{H}_{\mathrm{R}}$ depending on $\boldsymbol{H}_{\mathrm{R}}$ or $\boldsymbol{A}_{\mathrm{R}}$ alone. Likewise,

$$
\boldsymbol{E}_{\mathrm{E}}=M_{\mathrm{E}} * \boldsymbol{E}_{\mathrm{R}}=N_{\mathrm{E}} * A_{\mathrm{R}}
$$

for the elastic strain, with

$$
\begin{aligned}
M_{\mathrm{E}}^{\mathrm{T}}[\boldsymbol{a} \otimes \boldsymbol{c}] & :=-\frac{1}{2} \nabla_{\boldsymbol{c}} \boldsymbol{C}_{\mathrm{E}}\left[\nabla \boldsymbol{g}_{\boldsymbol{a}}\right]-\frac{1}{2} \nabla_{\boldsymbol{a}} \boldsymbol{C}_{\mathrm{E}}\left[\nabla \boldsymbol{g}_{\boldsymbol{c}}\right]-\delta \boldsymbol{a} \otimes \boldsymbol{c}, \\
\boldsymbol{N}_{\mathrm{E}}^{\mathrm{T}}[\boldsymbol{a} \otimes \boldsymbol{c}] & :=\frac{1}{2} \boldsymbol{C}_{\mathrm{E}}\left[\nabla \boldsymbol{g}_{\boldsymbol{a}}\right] \operatorname{axt} \boldsymbol{c}+\frac{1}{2} \boldsymbol{C}_{\mathrm{E}}\left[\nabla \boldsymbol{g}_{\boldsymbol{c}}\right] \mathrm{axt} \boldsymbol{a} .
\end{aligned}
$$

Since both relations (A.14) for $\boldsymbol{E}_{\mathrm{E}}$ have been derived from the same starting relation (A.5), they are clearly completely equivalent.

\section{Appendix A.2. Spectral formulation}

As usual, in the current context of periodic fields, Fourier transform methods (e.g., Khachaturyan, 1983; Mura, 1987; Suquet, 1997; Nemat-Nasser and Hori, 1999; Li and Wang, 2008) are employed. In particular, the Fourier transform of the gradient of (A.1) yields the result $\hat{\boldsymbol{H}}(\boldsymbol{k})=$ $-\hat{\boldsymbol{G}}_{\mathrm{E}}(\boldsymbol{k}) \hat{\boldsymbol{T}}_{\mathrm{R}}(\boldsymbol{k})(\boldsymbol{k} \otimes \boldsymbol{k})$ for the Fourier transform $\hat{\boldsymbol{H}}$ of $\boldsymbol{H}$. As usual, $\hat{\boldsymbol{G}}_{\mathrm{E}}$ is determined by the Fourier transform $\hat{\boldsymbol{K}}_{\mathrm{E}}$ of the acoustic tensor $\boldsymbol{K}_{\mathrm{E}}=\boldsymbol{G}_{\mathrm{E}}^{-1}$, defined by $\boldsymbol{a} \cdot \hat{\boldsymbol{K}}_{\mathrm{E}} \boldsymbol{b}:=\boldsymbol{a} \otimes \boldsymbol{k} \cdot \boldsymbol{C}_{\mathrm{E}}[\boldsymbol{b} \otimes \boldsymbol{k}]=\boldsymbol{a} \cdot \boldsymbol{C}_{\mathrm{E}}[\boldsymbol{b} \otimes \boldsymbol{k}] \boldsymbol{k}$ 
in terms of $\boldsymbol{C}_{\mathrm{E}}$ and arbitrary $\boldsymbol{a}, \boldsymbol{b}$. Since $\hat{\boldsymbol{K}}_{\mathrm{E}}(\mathbf{0})=\mathbf{0}, \hat{\boldsymbol{G}}_{\mathrm{E}}(\boldsymbol{k})$, and so $\hat{\boldsymbol{H}}(\boldsymbol{k})$, are defined only for $\boldsymbol{k} \neq \mathbf{0}$. On this basis,

$$
\hat{\boldsymbol{E}}_{\mathrm{E}}(\boldsymbol{k})=\hat{\boldsymbol{M}}_{\mathrm{E}}(\boldsymbol{k}) \hat{\boldsymbol{E}}_{\mathrm{R}}(\boldsymbol{k})=\left\{\hat{\boldsymbol{\Gamma}}_{\mathrm{E}}(\boldsymbol{k}) \boldsymbol{C}_{\mathrm{E}}-\boldsymbol{I}\right\} \hat{\boldsymbol{E}}_{\mathrm{R}}(\boldsymbol{k}),
$$

follows from (A.14) for the elastic strain depending on $\hat{\boldsymbol{E}}_{\mathrm{R}}$ and determined by the LippmannSchwinger operator $\boldsymbol{\Gamma}_{\mathrm{E}}$ (e.g., Suquet, 1997), with

$$
\hat{\boldsymbol{\Gamma}}_{\mathrm{E}}(\boldsymbol{k}) \boldsymbol{Z}:=\operatorname{sym}\left\{\hat{\boldsymbol{G}}_{\mathrm{E}}(\boldsymbol{k}) \boldsymbol{Z}(\boldsymbol{k} \otimes \boldsymbol{k})\right\}
$$

for any second-order tensor $\boldsymbol{Z}$. Given (A.16),

$$
\hat{\boldsymbol{T}}(\boldsymbol{k})=\left\{\boldsymbol{C}_{\mathrm{E}} \hat{\boldsymbol{\Gamma}}_{\mathrm{E}}(\boldsymbol{k}) \boldsymbol{C}_{\mathrm{E}}-C_{\mathrm{E}}\right\} \hat{\boldsymbol{E}}_{\mathrm{R}}(\boldsymbol{k}),
$$

follows the stress from (11), again for $\boldsymbol{k} \neq \mathbf{0}$. For $\boldsymbol{k}=\mathbf{0}$,

$$
\hat{\boldsymbol{T}}(\mathbf{0})=C_{\mathrm{E}} \hat{\boldsymbol{E}}_{\mathrm{E}}(\mathbf{0}), \quad \hat{\boldsymbol{E}}_{\mathrm{E}}(\mathbf{0})=S_{\mathrm{E}} \hat{\boldsymbol{T}}(\mathbf{0})=C_{\mathrm{E}}^{-1} \hat{\boldsymbol{T}}(\mathbf{0}),
$$

hold directly from (11). Lastly, the relation

$$
\begin{aligned}
\Psi_{\text {ela }} & :=\int \psi_{\text {ela }}(\boldsymbol{x}) d v(\boldsymbol{x}) \\
& =\frac{1}{(2 \pi)^{3}} \int \frac{1}{2} \hat{\boldsymbol{E}}_{\mathrm{E}}(\boldsymbol{k}) \cdot \boldsymbol{C}_{\mathrm{E}} \hat{\boldsymbol{E}}_{\mathrm{E} *}(\boldsymbol{k}) d v(\boldsymbol{k}) \\
& =\frac{1}{2} \overline{\boldsymbol{E}}_{\mathrm{E}} \cdot \boldsymbol{C}_{\mathrm{E}} \overline{\boldsymbol{E}}_{\mathrm{E}}+\frac{1}{(2 \pi)^{3}} f \frac{1}{2} \hat{\boldsymbol{E}}_{\mathrm{E}}(\boldsymbol{k}) \cdot \boldsymbol{C}_{\mathrm{E}} \hat{\boldsymbol{E}}_{\mathrm{E} *}(\boldsymbol{k}) d v(\boldsymbol{k})
\end{aligned}
$$

for the elastic energy is obtained from

$$
\psi_{\text {ela }}=\frac{1}{2} \boldsymbol{E}_{\mathrm{E}} \cdot \boldsymbol{C}_{\mathrm{E}} \boldsymbol{E}_{\mathrm{E}}
$$

via the first relation in (6), inverse Fourier transformation, the constancy of $C_{\mathrm{E}}$, and the RayleighPlancherel theorem (e.g., Bracewell, 2000, pp. 119-120). Here, $\boldsymbol{E}_{\mathrm{E}}=\boldsymbol{E}-\boldsymbol{E}_{\mathrm{R}}$ is the elastic strain, $\bar{f} \equiv \hat{f}(\mathbf{0})$ is the average value of $f(\boldsymbol{x})$ over the periodic unit cell, and $\hat{f}_{*}(\boldsymbol{k})=\hat{f}(-\boldsymbol{k})$ represents the complex conjugate of $\hat{f}(\boldsymbol{k})$. Using (A.16),

$$
\hat{\boldsymbol{E}}_{\mathrm{E}} \cdot \boldsymbol{C}_{\mathrm{E}} \hat{\boldsymbol{E}}_{\mathrm{E} *}=\hat{\boldsymbol{E}}_{\mathrm{R}} \cdot \hat{\boldsymbol{A}}_{\mathrm{E}} \hat{\boldsymbol{E}}_{\mathrm{R} *}
$$

follows with

$$
\hat{A}_{\mathrm{E}}:=\left(C_{\mathrm{E}} \hat{\Gamma}_{\mathrm{E}}-I\right) C_{\mathrm{E}}\left(\hat{\Gamma}_{\mathrm{E} *} C_{\mathrm{E}}-I\right) .
$$

Note that $\boldsymbol{A}_{\mathrm{E}}$ is equivalent to the operator $\boldsymbol{A} \equiv A_{\text {mпи }} \boldsymbol{i}_{m} \otimes \boldsymbol{i}_{n} \otimes \boldsymbol{i}_{u} \otimes \boldsymbol{i}_{v}$ in Hunter et al. $(2011,2013)$. Substituting (A.22) into (A.20) yields

$$
\Psi_{\text {ela }}=\frac{1}{2} \overline{\boldsymbol{T}} \cdot \boldsymbol{S}_{\mathrm{E}} \overline{\boldsymbol{T}}+\frac{1}{(2 \pi)^{3}} f \frac{1}{2} \hat{\boldsymbol{E}}_{\mathrm{R}}(\boldsymbol{k}) \cdot \hat{\boldsymbol{A}}_{\mathrm{E}}(\boldsymbol{k}) \hat{\boldsymbol{E}}_{\mathrm{R} *}(\boldsymbol{k}) d v(\boldsymbol{k})
$$

in terms of $\hat{\boldsymbol{E}}_{\mathrm{R}}$ for the case of stress control with $\overline{\boldsymbol{T}} \equiv \hat{\boldsymbol{T}}(\mathbf{0})$ the mean (far-field) stress. Subtracting the "potential energy" $(2 \pi)^{3} \overline{\boldsymbol{T}} \cdot \overline{\boldsymbol{E}}$ of stress control from (A.24) results in the elastic energy model employed by Hunter et al. $(2011,2013)$ and Mianroodi and Svendsen (2015). 


\section{Appendix B. Generalized PN model of Xiang et al. (2008)}

As already mentioned above, the generalized PN model of Xiang et al. (2008) is based in particular on (A.14) for $\boldsymbol{E}_{\mathrm{E}}$. They considered slip with respect to the (111) plane (e.g., $(x, y)$ plane at $z=0)$ parameterized by phase or disregistry fields $\phi(x, y)$ in the [110] direction, $\psi(x, y)$ in the [1̄12] direction (analogous to e.g. Bulatov and Cai, 2006; Mianroodi and Svendsen, 2015), as well as one $\eta(x, y)$ in the [111] direction normal to (111). In terms of these and the corresponding basis system $\left(\boldsymbol{i}_{x}, \boldsymbol{i}_{y}, \boldsymbol{i}_{z}\right)=([\overline{1} 10] / \sqrt{2},[\overline{1} \overline{1} 2] / \sqrt{6},[111] / \sqrt{3})$,

$$
\boldsymbol{H}_{\mathrm{R}}(x, y, z)=\phi(x, y) \delta(z) \boldsymbol{i}_{x} \otimes \boldsymbol{i}_{z}+\psi(x, y) \delta(z) \boldsymbol{i}_{y} \otimes \boldsymbol{i}_{z}+\eta(x, y) \delta(z) \boldsymbol{i}_{z} \otimes \boldsymbol{i}_{z},
$$

represents the residual distortion model of Xiang et al. (2008). In turn, this implies the form ${ }^{2}$

$$
\begin{aligned}
\boldsymbol{A}_{\mathrm{R}}(x, y, z) & =\phi_{y}(x, y) \delta(z) \boldsymbol{i}_{x} \otimes \boldsymbol{i}_{x}+\psi_{y}(x, y) \delta(z) \boldsymbol{i}_{y} \otimes \boldsymbol{i}_{x}+\eta_{y}(x, y) \delta(z) \boldsymbol{i}_{z} \otimes \boldsymbol{i}_{x} \\
& -\phi_{x}(x, y) \delta(z) \boldsymbol{i}_{x} \otimes \boldsymbol{i}_{y}-\psi_{x}(x, y) \delta(z) \boldsymbol{i}_{y} \otimes \boldsymbol{i}_{y}-\eta_{x}(x, y) \delta(z) \boldsymbol{i}_{z} \otimes \boldsymbol{i}_{y},
\end{aligned}
$$

for the dislocation tensor. Here, $f_{x}:=f_{, x}$ and $f_{y}:=f_{, y}$. As just established above, the model for elastic energy and stress assumed by Xiang et al. (2008) is equivalent that employed by Hunter et al. (2011, 2013) and Mianroodi and Svendsen (2015). Consequently, (A.18) for $\hat{\boldsymbol{T}}$ in terms of $\hat{\boldsymbol{E}}_{\mathrm{R}}$ holds in the model of Xiang et al. (2008) as well. Given the Fourier transform

$$
\hat{\boldsymbol{H}}_{\mathrm{R}}\left(k_{x}, k_{y}, k_{z}\right)=\hat{\phi}\left(k_{x}, k_{y}\right) \boldsymbol{i}_{x} \otimes \boldsymbol{i}_{z}+\hat{\psi}\left(k_{x}, k_{y}\right) \boldsymbol{i}_{y} \otimes \boldsymbol{i}_{z}+\hat{\eta}\left(k_{x}, k_{y}\right) \boldsymbol{i}_{z} \otimes \boldsymbol{i}_{z}
$$

of (B.1), one obtains

$$
\begin{aligned}
\hat{\boldsymbol{T}}\left(k_{x}, k_{y}, k_{z}\right) & =\hat{\phi}\left(k_{x}, k_{y}\right) \hat{\boldsymbol{M}}_{\mathrm{E} x}\left(k_{x}, k_{y}, k_{z}\right)+\hat{\psi}\left(k_{x}, k_{y}\right) \hat{\boldsymbol{M}}_{\mathrm{E} y}\left(k_{x}, k_{y}, k_{z}\right) \\
& +\hat{\eta}\left(k_{x}, k_{y}\right) \hat{\boldsymbol{M}}_{\mathrm{E} z}\left(k_{x}, k_{y}, k_{z}\right)
\end{aligned}
$$

with

$$
\hat{\boldsymbol{M}}_{\mathrm{E} a}:=\left(\boldsymbol{C}_{\mathrm{E}} \hat{\boldsymbol{\Gamma}}_{\mathrm{E}} C_{\mathrm{E}}-C_{\mathrm{E}}\right)\left[\boldsymbol{i}_{a} \otimes i_{z}\right]
$$

in terms of $\hat{\boldsymbol{\Gamma}}_{\mathrm{E}}$ from (A.17). Lastly, partial inverse transformation

$$
\hat{\boldsymbol{T}}\left(k_{x}, k_{y}\right):=\hat{\boldsymbol{T}}\left(k_{x}, k_{y}, z=0\right)=\frac{1}{2 \pi} \int_{\mathbb{R}} \hat{\boldsymbol{T}}\left(k_{x}, k_{y}, k_{z}\right) d k_{z}
$$

results in the reduced stress (Xiang et al., 2008, Equation (12))

$$
\hat{\boldsymbol{T}}\left(k_{x}, k_{y}\right)=\hat{\phi}\left(k_{x}, k_{y}\right) \hat{\boldsymbol{M}}_{\mathrm{E} x}\left(k_{x}, k_{y}\right)+\hat{\psi}\left(k_{x}, k_{y}\right) \hat{\boldsymbol{M}}_{\mathrm{E} y}\left(k_{x}, k_{y}\right)+\hat{\eta}\left(k_{x}, k_{y}\right) \hat{\boldsymbol{M}}_{\mathrm{E} z}\left(k_{x}, k_{y}\right)
$$

(cf. Bulatov and Cai, 2006, Equation (11.55)), with now

$$
\hat{\boldsymbol{M}}_{\mathrm{E} a}\left(k_{x}, k_{y}\right):=\frac{1}{2 \pi} \int_{\mathbb{R}} \hat{\boldsymbol{M}}_{\mathrm{E} a}\left(k_{x}, k_{y}, k_{z}\right) d k_{z}
$$

(Xiang et al., 2008, Equations (8), (9) and (13)) for $a=x, y, z$.

\footnotetext{
${ }^{2}$ Following Mura (1987), Xiang et al. (2008) worked with the transpose of the definition (A.7) of curl $\boldsymbol{H}_{\mathrm{R}}$ used here.
} 


\section{Appendix C. Selected algorithmic details}

For completeness, a brief summary of the algorithmic formulation of the PF model of Mianroodi and Svendsen (2015) is provided here. This is based in particular on the split $\boldsymbol{u}=\boldsymbol{u}_{\mathrm{h}}+\boldsymbol{u}_{\mathrm{p}}$ of the displacement field into homogeneous $\boldsymbol{u}_{\mathrm{h}}=\overline{\boldsymbol{E}} \boldsymbol{x}$ and particular $\boldsymbol{u}_{\mathrm{p}}$ parts. In contrast to all previous algorithms (e.g., Moulinec and Suquet, 1994; Suquet, 1997; Dreyer and Müller, 2000; Brown et al., 2002; Monchiet and Bonnet, 2012), which are strain-based and rely in particular on various forms of the Lippmann-Schwinger operator $\boldsymbol{\Gamma}_{\mathrm{E}}$ from (A.17), the current algorithm is displacementbased. This is done for two reason. First of all, this facilitates comparison of atomic and continuum results at the more fundamental or elementary level of displacement. Secondly, it is numerically more efficient to do so. Indeed, the model expression

$$
\hat{\boldsymbol{u}}_{\mathrm{p}}(\boldsymbol{k})=\hat{\boldsymbol{G}}_{\mathrm{E}}(\boldsymbol{k}) \hat{\boldsymbol{T}}_{\mathrm{R}}(\boldsymbol{k}) \boldsymbol{\imath} \boldsymbol{k}
$$

from (A.1) for the particular part of the displacement field satisfying quasi-static momentum balance (10) depends only the second-order Green tensor $\hat{\boldsymbol{G}}_{\mathrm{E}}$; calculation of the fourth-order $\hat{\boldsymbol{\Gamma}}_{\mathrm{E}}$ is avoided completely.

The resulting algorithm is summarized in Algorithm 1.

1. $\bar{E}$ and $\phi$ given;

2. given $\boldsymbol{\phi}$, calculate $\boldsymbol{T}_{\mathrm{R}}$ from (A.2)

3. $\hat{\boldsymbol{T}}_{\mathrm{R}}=\mathcal{F}\left(\boldsymbol{T}_{\mathrm{R}}\right)$

4. $\hat{\boldsymbol{u}}_{\mathrm{p}}=\hat{\boldsymbol{G}}_{\mathrm{E}} \hat{\boldsymbol{T}}_{\mathrm{R}} \boldsymbol{l} \boldsymbol{k}$ from (A.1);

5. $\hat{\boldsymbol{E}}=\operatorname{sym}\left(\hat{\boldsymbol{u}}_{\mathrm{p}} \otimes \imath \boldsymbol{k}\right)$ for $\boldsymbol{k} \neq \mathbf{0}$;

6. $\hat{\boldsymbol{E}}=\overline{\boldsymbol{E}}$ for $\boldsymbol{k}=\mathbf{0}$

7. $\boldsymbol{E}=\mathcal{F}^{-1}(\hat{\boldsymbol{E}})$ and $\boldsymbol{u}_{\mathrm{p}}=\mathcal{F}^{-1}\left(\hat{\boldsymbol{u}}_{\mathrm{p}}\right)$

Algorithm 1: Displacement-based algorithm.

Note that the corresponding stress field

$$
\hat{\boldsymbol{T}}(\boldsymbol{k})= \begin{cases}C_{\mathrm{E}}\left[\hat{\boldsymbol{E}}(\boldsymbol{k})-\hat{\boldsymbol{E}}_{\mathrm{R}}(\boldsymbol{k})\right] & \boldsymbol{k} \neq \mathbf{0} \\ C_{\mathrm{E}}\left[\overline{\boldsymbol{E}}-\hat{\boldsymbol{E}}_{\mathrm{R}}(\mathbf{0})\right] & \boldsymbol{k}=\mathbf{0}\end{cases}
$$

is equivalent to the relation obtained for example by Bulatov and Cai (2006, Equation (11.55)). Also note that $\hat{\boldsymbol{E}}_{\mathrm{R}}(\mathbf{0})$ is determined by $\overline{\boldsymbol{\phi}}=\hat{\boldsymbol{\phi}}(\mathbf{0})$ representing the volume average of this over the unit cell.

Consider next the algorithm for numerical solution of the Ginzburg-Landau relation (12). Following Chen and Shen (1998), this is based semi-implicit time-integration of (12); in particular, first-order backward-difference approximation of $\dot{\phi}_{a}$, and first-order Adams-Bashforth approximation of $\partial_{\phi_{a}} \psi$, is employed. Let $I=\bigcup_{n=0}\left[t_{n}, t_{n+1}\right]$ represent the discretization of the time interval $I \subset \mathbb{R}$ of interest. For simplicity, assume that each subinterval $\left[t_{n}, t_{n+1}\right]$ of $I$ is of the same duration $\Delta t=t_{n+1}-t_{n}$. Consider an arbitrary subinterval $\left[t_{n}, t_{n+1}\right]$. Assume as usual that $\boldsymbol{E}_{n}, \boldsymbol{\phi}_{n}$ 
$\left(\phi:=\left(\phi_{1}, \ldots\right)\right)$, and so $\left(\partial_{\phi} \psi\right)_{n}:=\left(\partial_{\phi_{1}} \psi, \ldots\right)_{n}$, are known from the the solution obtained at the end of the previous time step. Fourier transformation of (12) and rearrangement then results in the linear system

$$
\hat{\mathbf{M}} \hat{\boldsymbol{\phi}}_{n+1}=\hat{\boldsymbol{\phi}}_{n}-m_{0} \Delta t \widehat{\left(\partial_{\boldsymbol{\phi}} \psi\right)_{n}}
$$

to solve for $\hat{\boldsymbol{\phi}}_{n+1}$ with $\hat{M}_{a b}:=\delta_{a b}+m_{0} \Delta t \psi_{\mathrm{G} 0} \hat{n}_{a b}^{g}$ and $\hat{n}_{a b}^{g}:=\boldsymbol{k} \cdot \boldsymbol{N}_{a b}^{g} \boldsymbol{k}$, with $\boldsymbol{N}_{a b}^{g}$ given by (9). Note that (C.3) is a direct update; no iteration is required. In addition, note that $\hat{\mathbf{M}}$ depends only on (constant) material data and the chosen discretization in wavenumber space. For a given material and discretization, then, it need be calculated only once at the start as part of the initial data.

As discussed in the text, all material properties $\psi_{\mathrm{H} 0}, \psi_{\mathrm{G} 0}, m_{0}$, as well as $\boldsymbol{H}_{a}$ and $\boldsymbol{N}_{a b}^{\phi}$ for $a, b=$ $1, \ldots, p$, are known and constant. In addition, this holds for $C_{\mathrm{E}}$ and so for $C_{\mathrm{H}}=C_{\mathrm{E}}$ determining $\boldsymbol{G}_{\mathrm{H}}$ and $\boldsymbol{\Gamma}_{\mathrm{H}}$. All field relations are solved in dimensionless form. The effective shear modulus $\mu_{\mathrm{fcc}}$ is used as the basic energy density scaling. Together with the (constant isothermal) mobility $m_{0}$ from (12), this determines the time scaling $1 /\left(\mu_{\mathrm{fcc}} m_{0}\right)$. Length scaling is based on $d_{111}$, which also represents the grid point spacing in the numerical simulations. In particular, in this case, the dislocation core is resolved over multiple grid points in the slip plane. 